\title{
SUBLATTICES OF LATTICES OF ORDER-CONVEX SETS, III. THE CASE OF TOTALLY ORDERED SETS
}

\author{
MARINA SEMENOVA AND FRIEDRICH WEHRUNG
}

\begin{abstract}
For a partially ordered set $P$, let $\mathbf{C o}(P)$ denote the lattice of all order-convex subsets of $P$. For a positive integer $n$, we denote by $\mathbf{S U B}(\mathcal{L O})$ (resp., SUB $(n)$ ) the class of all lattices that can be embedded into a lattice of the form

$$
\prod_{i \in I} \mathbf{C o}\left(T_{i}\right)
$$

where $\left\langle T_{i} \mid i \in I\right\rangle$ is a family of chains (resp., chains with at most $n$ elements). We prove the following results:

(1) Both classes $\mathbf{S U B}(\mathcal{L O})$ and $\mathbf{S U B}(n)$, for any positive integer $n$, are locally finite, finitely based varieties of lattices, and we find finite equational bases of these varieties.

(2) The variety $\mathbf{S U B}(\mathcal{L} \mathcal{O})$ is the quasivariety join of all the varieties $\mathbf{S U B}(n)$, for $1 \leq n<\omega$, and it has only countably many subvarieties. We classify these varieties, together with all the finite subdirectly irreducible members of $\mathbf{S U B}(\mathcal{L O})$.

(3) Every finite subdirectly irreducible member of $\mathbf{S U B}(\mathcal{L O})$ is projective within $\mathbf{S U B}(\mathcal{L O})$, and every subquasivariety of $\mathbf{S U B}(\mathcal{L O})$ is a variety.
\end{abstract}

\section{INTRODUCTION}

For a partially ordered set (from now on poset) $(P, \unlhd)$, a subset $X$ of $P$ is orderconvex, if $x \unlhd z \unlhd y$ and $\{x, y\} \subseteq X$ implies that $z \in X$, for all $x, y, z \in P$. The lattices of the form $\mathbf{C o}(P)$ have been characterized by G. Birkhoff and M. K. Bennett in 2]. In M. Semenova and F. Wehrung [12, the authors solve a problem stated in K. V. Adaricheva, V. A. Gorbunov, and V. I. Tumanov [1], by proving the following result.

Theorem 1. The class SUB of all lattices that can be embedded into some lattice of the form $\mathbf{C o}(P)$ forms a variety, defined by three identities, (S), (U), and (B).

In M. Semenova and F. Wehrung [13, this result is extended to special classes of posets $P$ :

Theorem 2. For a positive integer $n$, the class $\mathbf{S} \mathbf{U B}_{n}$ of all lattices that can be embedded into some lattice of the form $\mathbf{C o}(P)$, where $P$ is a poset of length at

Date: November 13, 2018.

2000 Mathematics Subject Classification. Primary: 06B05, 06B20, 06B15, 06A05, 08C15. Secondary: 05B25.

Key words and phrases. Lattice, embedding, poset, chain, order-convex, variety, join-irreducible, join-seed.

The first author was partially supported by INTAS grant no. YSF: 2001/1-65. The authors were partially supported by GA CR grant no. 201/00/0766 and by institutional grant MSM:J13/98:1132000007a. 
most $n$, is a variety, defined by the identities (S), (U), (B), together with new identities $\left(\mathrm{H}_{n}\right)$ and $\left(\mathrm{H}_{k, n+1-k}\right)$, for $1 \leq k \leq n$.

In the present paper, we extend these results to sublattices of products of lattices of convex subsets of chains (i.e., totally ordered sets), thus solving a problem of 12 . More specifically, we denote by $\mathbf{S U B}(\mathcal{L O})$ (resp., $\mathbf{S U B}(n)$ ) the class of all lattices that can be embedded into a lattice of the form

$$
\prod_{i \in I} \mathbf{C o}\left(T_{i}\right),
$$

where $\left\langle T_{i} \mid i \in I\right\rangle$ is a family of chains (resp., chains with at most $n$ elements). We prove the following results:

(1) Both classes $\mathbf{S U B}(\mathcal{L O})$ and $\mathbf{S U B}(n)$ are finitely based varieties of lattices, for any positive integer $n$. Moreover, $\mathbf{S U B}(n+1)=\mathbf{S U B}(\mathcal{L} \mathcal{O}) \cap \mathbf{S U B}_{n}$ (Theorems 8.2 and 9.4).

(2) By using a result of V. Slavík [14, we prove that the variety $\mathbf{S U B}(\mathcal{L O})$ is locally finite (Theorem 9.5).

(3) The variety $\mathbf{S U B}(\mathcal{L O})$ is the quasivariety join of all the varieties $\mathbf{S U B}(n)$, for $1 \leq n<\omega$ (Corollary 9.7), and every proper subvariety of $\mathbf{S U B}(\mathcal{L O})$ is finitely generated (Corollary 11.7).

(4) The only proper subvarieties of $\mathbf{S U B}(\mathcal{L O})$ are those between $\mathbf{S U B}(n)$ and SUB $(n+1)$ for some natural number $n$ (Theorem 11.5).

(5) We classify all finite subdirectly irreducible members of $\mathbf{S U B}(\mathcal{L O})$, and we describe exactly the lattice of all subvarieties of $\mathbf{S U B}(\mathcal{L O})$ (Theorem 11.5 to Corollary [1.9.

(6) All finite subdirectly irreducible members of $\mathbf{S U B}(\mathcal{L O})$ are projective within $\mathbf{S U B}(\mathcal{L O})$ (Theorem[12.4), and every subquasivariety of $\mathbf{S U B}(\mathcal{L O})$ is a variety (Theorem 12.5).

The main technical result towards the proof that $\mathbf{S U B}(\mathcal{L O})$ is a variety is that the reflexive closure of the join-dependency relation $D$ is transitive, in any member of $\mathbf{S U B}(\mathcal{L O})$ with 'enough' join-irreducible elements (Corollary 6.2). This may be viewed as an analogue, for certain join-semidistributive lattices, of the transitivity of perspectivity proved by von Neumann in continuous geometries, see [11].

We refer the reader to our papers [12, 13] for unexplained notation and terminology. In particular, the identities $(\mathrm{S}),(\mathrm{U})$, and $(\mathrm{B})$, together with their join-irreducible translations $\left(\mathrm{S}_{\mathrm{j}}\right),\left(\mathrm{U}_{\mathrm{j}}\right)$, and $\left(\mathrm{B}_{\mathrm{j}}\right)$, and tools such as Stirlitz tracks or the Udav-Bond partition, are defined in [12]. The identities $\left(\mathrm{H}_{n}\right)$ and $\left(\mathrm{H}_{m, n}\right)$, their join-irreducible translations, and bi-Stirlitz tracks are defined in 13. We shall often use the trivial fact that $\mathbf{C o}(P, \unlhd)=\mathbf{C o}(P, \unrhd)$, for any poset $(P, \unlhd)$, where $\unrhd$ denotes the converse order of $\unlhd$.

The join-dependency relation on a lattice $L$, see R. Freese, J. Ježek, and J. B. Nation [5], is defined on the set $\mathrm{J}(L)$ of all join-irreducible elements of $L$, and it is written $D_{L}$, or $D$ if $L$ is understood from the context. For $a \in \mathrm{J}(L)$, we write, as in 12 13,

$$
[a]^{D}=\{x \in \mathrm{J}(L) \mid a D x\} .
$$

\section{JOIN-SEEDS AND MORE MINIMAL COVERS}

We recall from [13] the following definition: 
Definition 2.1. A subset $\Sigma$ of a lattice $L$ is a join-seed, if the following statements hold:

(i) $\Sigma \subseteq \mathrm{J}(L)$;

(ii) every element of $L$ is a join of elements of $\Sigma$;

(iii) for all $p \in \Sigma$ and all $a, b \in L$ such that $p \leq a \vee b$ and $p \not \leq a, b$, there are $x \leq a$ and $y \leq b$ both in $\Sigma$ such that $p \leq x \vee y$ is minimal in $x$ and $y$.

Two important examples of join-seeds are provided by the following lemma, see [13].

Lemma 2.2. Any of the following assumptions implies that the subset $\Sigma$ is a joinseed of the lattice $L$ :

(i) $L=\mathbf{C o}(P)$ and $\Sigma=\{\{p\} \mid p \in P\}$, for some poset $P$.

(ii) $L$ is a dually 2-distributive, complete, lower continuous, finitely spatial lattice, and $\Sigma=\mathrm{J}(L)$.

Lemma 2.3. Let $L$ be a lattice satisfying (B), let $\Sigma$ be a join-seed of $L$, let $p \in \Sigma$, let $x, y \in[p]^{D}$. If the inequality $p \leq x \vee y$ holds, then it is minimal in both $x$ and $y$.

Proof. From the assumption that $x, y \in[p]^{D}$, it follows that $p \not \leq x, y$. Since $p \leq x \vee y$ and $\Sigma$ is a join-seed of $L$, there are $u \leq x$ and $v \leq y$ in $\Sigma$ such that the inequality $p \leq u \vee v$ holds and is minimal in both $u$ and $v$. Furthermore, by the definition of the $D$ relation and since $\Sigma$ is a join-seed of $L$, there are $x^{\prime}, y^{\prime} \in \Sigma$ such that both inequalities $p \leq x \vee x^{\prime}$ and $p \leq y \vee y^{\prime}$ hold and are minimal in $x, x^{\prime}$, $y, y^{\prime}$. By applying $\left(\mathrm{B}_{\mathrm{j}}\right)$ to the inequalities $p \leq x \vee x^{\prime}, u \vee v$ and by observing that $p \not \leq x, v$, we obtain that $p \leq x^{\prime} \vee u$. Since $u \leq x$ and the inequality $p \leq x \vee x^{\prime}$ is minimal in $x$, we obtain that $u=x$. Similarly, $v=y$.

Lemma 2.4. Let $L$ be a lattice satisfying (B), let $\Sigma$ be a join-seed of $L$. Then $[p]^{D} \cap \Sigma$ is an antichain of $L$, for any $p \in \Sigma$.

Proof. Let $x, y \in[p]^{D}$. Since $\Sigma$ is a join-seed of $L$, there are $x^{\prime}, y^{\prime} \in \Sigma$ such that both inequalities $p \leq x \vee x^{\prime}$ and $p \leq y \vee y^{\prime}$ are minimal nontrivial join-covers. Observe that $p \not \leq x, x^{\prime}, y, y^{\prime}$. If $x \leq y$, then, since $p \not \leq y=x \vee y$ and $L$ satisfies $\left(\mathrm{B}_{\mathrm{j}}\right)$, the inequality $p \leq x \vee y^{\prime}$ holds. Since $x \leq y$ and the inequality $p \leq y \vee y^{\prime}$ is minimal in $y$, we obtain that $x=y$.

\section{The IDEnTity (E)}

Let (E) be the following identity in the variables $x, a, b_{0}, b_{1}, b_{2}$ :

$$
\begin{aligned}
x \wedge \bigwedge_{i<3}\left(a \vee b_{i}\right)=\bigvee_{i<3}\left[x \wedge b_{i} \wedge \bigwedge_{j \neq i}\left(a \vee b_{j}\right)\right] & \\
& \vee \bigvee_{\sigma \in \mathfrak{S}_{3}}\left[x \wedge\left(a \vee b_{0, \sigma}^{*}\right) \wedge\left(a \vee b_{1, \sigma}^{*}\right) \wedge\left(a \vee b_{\sigma(2)}\right)\right],
\end{aligned}
$$

where we denote by $\mathfrak{S}_{3}$ the group of all permutations of $\{0,1,2\}$ and we put

$$
\begin{aligned}
& b_{0, \sigma}^{*}=b_{\sigma(0)} \wedge\left(x \vee b_{\sigma(1)}\right), \\
& b_{1, \sigma}^{*}=b_{\sigma(1)} \wedge\left(x \vee b_{\sigma(2)}\right) \wedge\left(b_{\sigma(0)} \vee b_{\sigma(2)}\right),
\end{aligned}
$$

for all $\sigma \in \mathfrak{S}_{3}$. 
We now introduce a lattice-theoretical axiom, the join-irreducible interpretation of $(\mathrm{E})$, that we will denote by $\left(\mathrm{E}^{\Sigma}\right)$.

Definition 3.1. For a lattice $L$ and a subset $\Sigma$ of $\mathrm{J}(L)$, we say that $L$ satisfies $\left(\mathrm{E}^{\Sigma}\right)$, if for all elements $x, a, b_{0}, b_{1}$, and $b_{2}$ of $\Sigma$, if the inequality $x \leq a \vee b_{i}$ is a minimal nontrivial join-cover, for every $i<3$, then there exists $\sigma \in \mathfrak{S}_{3}$ such that $b_{\sigma(0)} \leq x \vee b_{\sigma(1)} \leq x \vee b_{\sigma(2)}$ and $b_{\sigma(1)} \leq b_{\sigma(0)} \vee b_{\sigma(2)}$.

The geometrical meaning of $\left(\mathrm{E}^{\Sigma}\right)$ is illustrated on Figure 1. The lines of that figure represent the ordering of the either the poset $P$ or its dual (and not the ordering of $L)$ in case $L=\mathbf{C o}(P, \unlhd)$. For example, the left half of Figure 1 represents (up to dualization of $\unlhd$ ) the relations $a \unlhd x \unlhd b_{i}$, for $i<3$, so that the inequality $\{x\} \leq\{a\} \vee\left\{b_{i}\right\}$ holds in $L$. Similar conventions hold for Figures 2 and 3 .

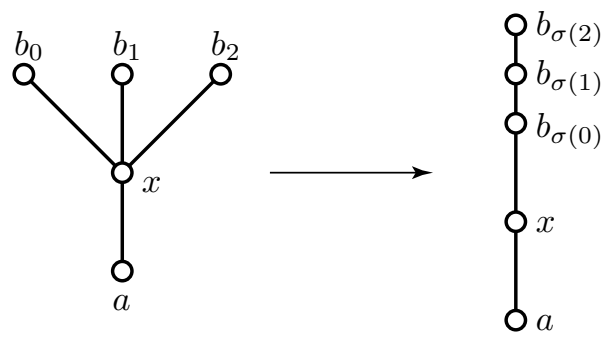

FigURE 1. Illustrating $\left(\mathrm{E}^{\Sigma}\right)$

Lemma 3.2. Let $L$ be a lattice, let $\Sigma$ be a subset of $\mathrm{J}(L)$. Then the following statements hold:

(i) If L satisfies (E), then L satisfies $\left(\mathrm{E}^{\Sigma}\right)$.

(ii) If $\Sigma$ is a join-seed of $L$ and $L$ satisfies both (B) and $\left(\mathrm{E}^{\Sigma}\right)$, then $L$ satisfies (E).

Proof. (i) Suppose that $x, a, b_{0}, b_{1}, b_{2} \in \Sigma$ satisfy the premise of $\left(\mathrm{E}^{\Sigma}\right)$. Since $x$ is join-irreducible and $x \not \leq b_{i}$, for all $i<3$, we obtain, by applying the identity (E) and using the notation introduced in (3.1) and (3.2), that there exists $\sigma \in \mathfrak{S}_{3}$ such that both inequalities $x \leq a \vee b_{0, \sigma}^{*}, a \vee b_{1, \sigma}^{*}$ hold. Since $b_{i, \sigma}^{*} \leq b_{\sigma(i)}$, it follows from the minimality of $b_{\sigma(i)}$ in the inequality $x \leq a \vee b_{\sigma(i)}$ that $b_{i, \sigma}^{*}=b_{\sigma(i)}$, for all $i<2$. Therefore, $b_{\sigma(0)} \leq x \vee b_{\sigma(1)} \leq x \vee b_{\sigma(2)}$ and $b_{\sigma(1)} \leq b_{\sigma(0)} \vee b_{\sigma(2)}$.

(ii) Let $c$ (resp., $d$ ) denote the left hand side (resp., right hand side) of the identity (E). Since $d \leq c$ holds in any lattice, it suffices to prove that $c \leq d$. Let $p \in \Sigma$ with $p \leq c$, we prove that $p \leq d$. If $p \leq a$, then $p \leq x \wedge a \leq d$. If $p \leq b_{i}$, for some $i<3$, then $p \leq x \wedge b_{i} \wedge \bigwedge_{j \neq i}\left(a \vee b_{j}\right) \leq d$.

Suppose from now on that $p \not \leq a$ and $p \not \leq b_{i}$, for all $i<3$. Since $p \leq a \vee b_{i}$ and $\Sigma$ is a join-seed of $L$, there are $u_{i} \leq a$ and $v_{i} \leq b_{i}$ in $\Sigma$ such that the inequality $p \leq u_{i} \vee v_{i}$ is a minimal nontrivial join-cover, for all $i<3$. In particular, $u_{i}$, $v_{i} \in[p]^{D}$. Put $u=u_{0}$, and let $i<3$. By applying $\left(\mathrm{B}_{\mathrm{j}}\right)$ to the inequalities $p \leq u \vee v_{0}, u_{i} \vee v_{i}$ and observing that $p \not \leq a$ (thus $p \not \leq u \vee u_{i}$ ), we obtain the inequality $p \leq u \vee v_{i}$. Furthermore, by Lemma 2.3 this inequality is minimal in both $u$ and $v_{i}$. Hence, by $\left(\mathrm{E}^{\Sigma}\right)$, there exists $\sigma \in \mathfrak{S}_{3}$ such that $v_{\sigma(0)} \leq p \vee v_{\sigma(1)} \leq p \vee v_{\sigma(2)}$ and 
$v_{\sigma(1)} \leq v_{\sigma(0)} \vee v_{\sigma(2)}$. Therefore, by putting

$$
\begin{aligned}
& v_{0, \sigma}^{*}=v_{\sigma(0)} \wedge\left(p \vee v_{\sigma(1)}\right), \\
& v_{1, \sigma}^{*}=v_{\sigma(1)} \wedge\left(p \vee v_{\sigma(2)}\right) \wedge\left(v_{\sigma(0)} \vee v_{\sigma(2)}\right),
\end{aligned}
$$

we obtain the equalities $v_{0, \sigma}^{*}=v_{\sigma(0)}$ and $v_{1, \sigma}^{*}=v_{\sigma(1)}$, and the inequalities

$$
p \leq x \wedge\left(u \vee v_{0, \sigma}^{*}\right) \wedge\left(u \vee v_{1, \sigma}^{*}\right) \wedge\left(u \vee v_{\sigma(2)}\right) \leq d
$$

Since every element of $L$ is a join of elements of $\Sigma$, the inequality $c \leq d$ follows.

Corollary 3.3. The lattice $\mathbf{C o}(T)$ satisfies the identity $(\mathrm{E})$, for any chain $(T, \unlhd)$.

Proof. We apply Lemma 3.2 to $L=\mathbf{C o}(T)$ together with the join-seed $\Sigma=$ $\{\{p\} \mid p \in T\}$. Let $x, a, b_{0}, b_{1}, b_{2} \in T$ such that the inequality $\{x\} \leq\{a\} \vee\left\{b_{i}\right\}$ is a minimal nontrivial join-cover, for all $i<3$. Since $\mathbf{C o}(T, \unlhd)=\mathbf{C o}(T, \unrhd)$, we may assume without loss of generality that $a \triangleleft x \triangleleft b_{0}$, thus $x \triangleleft b_{i}$, for all $i<3$. Since $T$ is a chain, there exists $\sigma \in \mathfrak{S}_{3}$ such that $b_{\sigma(0)} \unlhd b_{\sigma(1)} \unlhd b_{\sigma(2)}$, whence

$$
\left\{b_{\sigma(0)}\right\} \leq\{x\} \vee\left\{b_{\sigma(1)}\right\} \leq\{x\} \vee\left\{b_{\sigma(2)}\right\} \text { and }\left\{b_{\sigma(1)}\right\} \leq\left\{b_{\sigma(0)}\right\} \vee\left\{b_{\sigma(2)}\right\} .
$$

Hence $\mathbf{C o}(T)$ satisfies $\left(\mathrm{E}^{\Sigma}\right)$. Since $\mathbf{C o}(T)$ satisfies (B) (see [12]) and $\Sigma$ is a join-seed of $\mathbf{C o}(T)$, it follows from Lemma 3.2 that $\mathbf{C o}(T)$ satisfies $(\mathrm{E})$.

Lemma 3.4. Let $L$ be a join-semidistributive lattice satisfying the identity $(\mathrm{E})$, let $a, x \in \mathrm{J}(L)$ and $b_{0}, b_{1}, b_{2} \in \mathrm{J}(L)$ be distinct such that $x \leq a \vee b_{i}$ is a minimal nontrivial join-cover, for all $i<3$. Then $a \vee b_{0} \leq a \vee b_{1} \leq a \vee b_{2}$ implies that $a \vee b_{0}<a \vee b_{1}<a \vee b_{2}$ and $b_{1} \leq b_{0} \vee b_{2}$.

Proof. Let $i, j$ be distinct in $\{0,1,2\}$. If $a \vee b_{i}=a \vee b_{j}$, then, by the join-semidistributivity of $L, x \leq a \vee b_{i}=a \vee\left(b_{i} \wedge b_{j}\right)$; it follows from the minimality assumption on $b_{i}$ that $b_{i} \leq b_{j}$. Similarly, $b_{j} \leq b_{i}$, whence $b_{i}=b_{j}$, a contradiction. Thus we have obtained the inequalities

$$
a \vee b_{0}<a \vee b_{1}<a \vee b_{2} .
$$

On the other hand, it follows from Lemma 3.2 that there exists $\sigma \in \mathfrak{S}_{3}$ such that the inequalities

$$
\begin{gathered}
x \vee b_{\sigma(0)} \leq x \vee b_{\sigma(1)} \leq x \vee b_{\sigma(2)}, \\
b_{\sigma(1)} \leq b_{\sigma(0)} \vee b_{\sigma(2)}
\end{gathered}
$$

hold. From (3.4) it follows that $a \vee b_{\sigma(0)} \leq a \vee b_{\sigma(1)} \leq a \vee b_{\sigma(2)}$, thus, by (3.3), $\sigma$ is the identity. The conclusion follows from (3.4) and (3.5).

\section{The identity $(\mathrm{P})$}

Let $(\mathrm{P})$ be the following identity in the variables $a, b, c, d, b_{0}, b_{1}$ :

$$
\begin{aligned}
a \wedge\left(b^{\prime} \vee c\right) \wedge(c \vee d)= & \left(a \wedge b^{\prime} \wedge(c \vee d)\right) \vee\left(a \wedge d \wedge\left(b^{\prime} \vee c\right)\right) \\
& \vee\left[a \wedge\left(\left(b^{\prime} \wedge(a \vee d)\right) \vee c\right) \wedge(c \vee d)\right] \\
& \vee \bigvee_{i<2}\left[a \wedge\left(b_{i} \vee c\right) \wedge\left(\left(b^{\prime} \wedge\left(a \vee b_{i}\right) \wedge\left(b_{i} \vee d\right)\right) \vee c\right) \wedge(c \vee d)\right],
\end{aligned}
$$

where we put $b^{\prime}=b \wedge\left(b_{0} \vee b_{1}\right)$. 
We now introduce a lattice-theoretical axiom, the join-irreducible interpretation of $(\mathrm{P})$, that we will denote by $\left(\mathrm{P}^{\Sigma}\right)$.

Definition 4.1. For a lattice $L$ and a subset $\Sigma$ of $\mathrm{J}(L)$, we say that $L$ satisfies $\left(\mathrm{P}^{\Sigma}\right)$, if for all elements $a, b, c, d, b_{0}, b_{1}$ in $\Sigma$, if both inequalities $a \leq b \vee c, c \vee d$ are minimal nontrivial join-covers and $b \leq b_{0} \vee b_{1}$, then either $b \leq a \vee d$ or there exists $i<2$ such that $a \leq b_{i} \vee c$ and $b \leq a \vee b_{i}, b_{i} \vee d$.

The geometrical meaning of $\left(\mathrm{P}^{\Sigma}\right)$ is illustrated on Figure 2. Horizontal lines are meant to suggest that "no side is chosen yet". For example, the non-horizontal lines in the left half of Figure 2 represent various inequalities such as $c \unlhd a \unlhd d$ and $c \unlhd a \unlhd b$ (in case $L=\mathbf{C o}(P, \unlhd)$ ), while the horizontal line represents the inequalities $b_{1-i} \unlhd b \unlhd b_{i}$, for some $i<2$. A similar convention applies to Figure 3 .
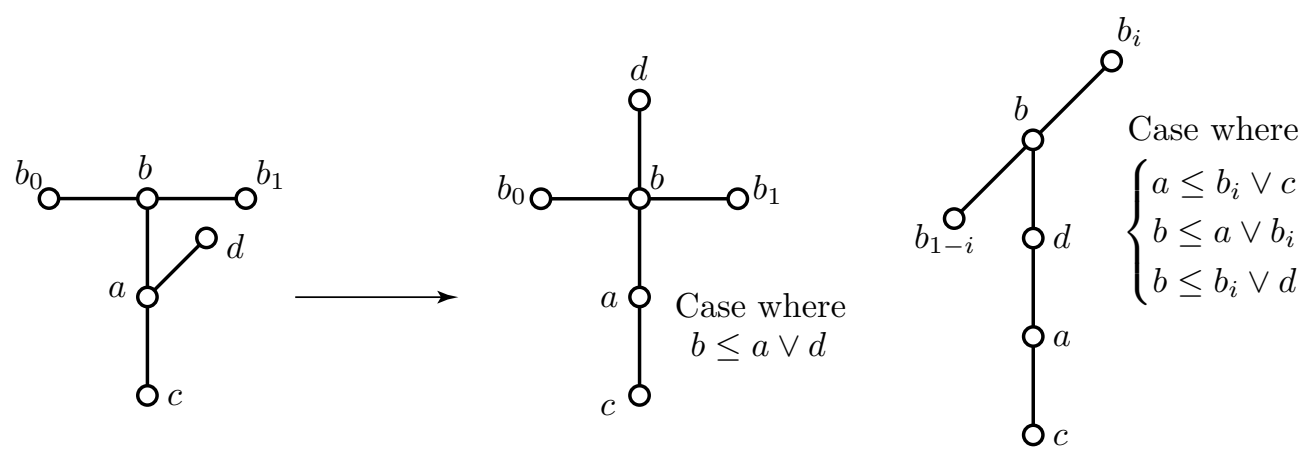

FiguRE 2. Illustrating $\left(\mathrm{P}^{\Sigma}\right)$

Lemma 4.2. Let $L$ be a lattice, let $\Sigma$ be a subset of $\mathrm{J}(L)$. Then the following statements hold:

(i) If $L$ satisfies $(\mathrm{P})$, then $L$ satisfies $\left(\mathrm{P}^{\Sigma}\right)$.

(ii) If $\Sigma$ is a join-seed of $L$ and $L$ satisfies both $(\mathrm{B})$ and $\left(\mathrm{P}^{\Sigma}\right)$, then $L$ satisfies $(\mathrm{P})$.

Proof. (i) Let $a, b, c, d, b_{0}, b_{1} \in \Sigma$ satisfy the premise of $\left(\mathrm{P}^{\Sigma}\right)$. Observe that $b \wedge\left(b_{0} \vee b_{1}\right)=b$, thus the left hand side of the identity (P) computed with these parameters equals $a$. Since $a \not \leq b, d$ and $a$ is join-irreducible, either $a \leq(b \wedge(a \vee d)) \vee c$ or $a \leq b_{i} \vee c$ and $a \leq\left(b \wedge\left(a \vee b_{i}\right) \wedge\left(b_{i} \vee d\right)\right) \vee c$, for some $i<2$. In the first case, from the fact that the cover $a \leq b \vee c$ is minimal in $b$ it follows that $b \leq a \vee d$ in the first case, and $b \leq a \vee b_{i}, b_{i} \vee d$ in the second case.

(ii) Let $e$ (resp., $f$ ) denote the left hand side (resp., right hand side) of the identity $(\mathrm{P})$. Let $p \in \Sigma$ such that $p \leq e$, we prove that $p \leq f$. If either $p \leq c$ or $p \leq b^{\prime}$ or $p \leq d$ this is obvious, so suppose, from now on, that $p \not \leq c, b^{\prime}, d$. Since $\Sigma$ is a join-seed of $L$, there are $u \leq b^{\prime}$ together with $v, v^{\prime} \leq c$ and $w \leq d$ in $\Sigma$ such that both inequalities

$$
\begin{aligned}
& p \leq u \vee v, \\
& p \leq v^{\prime} \vee w
\end{aligned}
$$


are minimal nontrivial join-covers. In particular, $u, v, v^{\prime}, w \in[p]^{D}$. Furthermore, by applying $\left(\mathrm{B}_{\mathrm{j}}\right)$ to the inequalities (4.1) and (4.2) and observing that $p \not \leq v \vee v^{\prime}$ (because $p \not c$ ), we obtain the inequality

$$
p \leq v \vee w .
$$

Furthermore, it follows from Lemma 2.3 that (4.3) is a minimal nontrivial joincover. Since $\Sigma$ is a join-seed of $L$, there are $u_{i} \leq b_{i}$ in $\Sigma \cup\{0\}$, for $i<2$, such that $u \leq u_{0} \vee u_{1}$. Suppose first that $u_{0}, u_{1} \in \Sigma$. Since $L$ satisfies $\left(\mathrm{P}^{\Sigma}\right)$, either

$$
u \leq p \vee w
$$

or

$$
p \leq u_{i} \vee v \text { and } u \leq p \vee u_{i}, u_{i} \vee w \text {, for some } i<2 \text {. }
$$

The conclusion (4.5) also holds if $u_{j}=0$, for some $j<2$, because $u \leq u_{1-j}$.

If (4.4) holds, then

$$
p \leq a \wedge((u \wedge(p \vee w)) \vee v) \wedge(v \vee w) \leq f
$$

If 4.5 holds, then

$$
p \leq a \wedge\left(u_{i} \vee v\right) \wedge\left(\left(u \wedge\left(p \vee u_{i}\right) \wedge\left(u_{i} \vee w\right)\right) \vee v\right) \wedge(v \vee w) \leq f .
$$

Since every element of $L$ is a join of elements of $\Sigma$, the inequality $e \leq f$ follows. Since $f \leq e$ holds in any lattice, we obtain that $e=f$.

Corollary 4.3. The lattice $\mathbf{C o}(T)$ satisfies $(\mathrm{P})$, for every chain $(T, \unlhd)$.

Proof. We apply Lemma 4.2 to $L=\mathbf{C o}(T)$ together with the join-seed $\Sigma=$ $\{\{p\} \mid p \in T\}$. Let $a, b, c, d, b_{0}, b_{1} \in T$ such that both inequalities $\{a\} \leq$ $\{b\} \vee\{c\},\{c\} \vee\{d\}$ are minimal nontrivial join-covers and $\{b\} \leq\left\{b_{0}\right\} \vee\left\{b_{1}\right\}$. Since $\mathbf{C o}(T, \unlhd)=\mathbf{C o}(T, \unrhd)$, we may assume without loss of generality that $c \triangleleft a \triangleleft b, d$. Furthermore, from $\{b\} \leq\left\{b_{0}\right\} \vee\left\{b_{1}\right\}$ it follows that there exists $i<2$ such that $b \unlhd b_{i}$. Since $T$ is a chain, either $b \unlhd d$ or $d \unlhd b$. In the first case, $\{b\} \leq\{a\} \vee\{d\}$. In the second case, $\{a\} \leq\left\{b_{i}\right\} \vee\{c\}$ and $\{b\} \leq\{a\} \vee\left\{b_{i}\right\},\left\{b_{i}\right\} \vee\{d\}$.

Hence $\mathbf{C o}(T)$ satisfies $\left(\mathrm{P}^{\Sigma}\right)$. By Lemma 4.2, $\mathbf{C o}(T)$ satisfies $(\mathrm{P})$.

\section{The IDEnTITy (HS)}

Let (HS) be the following identity in the variables $a, b, c, b_{0}, b_{1}$ :

$$
\begin{aligned}
a \wedge\left(b^{\prime} \vee c\right)= & \left(a \wedge b^{\prime}\right) \vee \bigvee_{i<2}\left[a \wedge\left(\left(b \wedge b_{i}\right) \vee c\right)\right] \\
& \vee \bigvee_{i<2}\left[a \wedge\left(\left(b^{\prime} \wedge\left(a \vee b_{i}\right)\right) \vee c\right) \wedge\left(b_{i} \vee c\right) \wedge\left(b \vee b_{1-i}\right)\right] \\
& \vee \bigvee_{i<2}\left[a \wedge\left(\left(b^{\prime} \wedge\left(a \vee b_{i}\right)\right) \vee c\right) \wedge\left(b_{0} \vee c\right) \wedge\left(b_{1} \vee c\right)\right],
\end{aligned}
$$

where we put $b^{\prime}=b \wedge\left(b_{0} \vee b_{1}\right)$. Since the right hand side of (HS) lies obviously below the right hand side of the identity $(S)$ while the left hand sides are the same, we obtain immediately the following result.

Lemma 5.1. The identity (HS) implies the Stirlitz identity (S). 
As observed in [12, (S) implies both join-semidistributivity and dual 2-distributivity. Therefore, we obtain the following consequence.

Lemma 5.2. The identity (HS) implies both join-semidistributivity and dual 2-distributivity.

We now introduce a lattice-theoretical axiom, the join-irreducible interpretation of (HS), that we will denote by $\left(\mathrm{HS}^{\Sigma}\right)$.

Definition 5.3. For a lattice $L$ and a subset $\Sigma$ of $\mathrm{J}(L)$, we say that $L$ satisfies $\left(\mathrm{HS}^{\Sigma}\right)$, if for all elements $a, b, c, b_{0}, b_{1}$ in $\Sigma$, if $a \neq b$, the inequality $a \leq b \vee c$ is minimal in $b$, and $b \leq b_{0} \vee b_{1}$ is a nontrivial join-cover, then there exists $i<2$ such that $b \leq a \vee b_{i}$ and either $a \leq b_{i} \vee c, b \vee b_{1-i}$ or $a \leq b_{0} \vee c, b_{1} \vee c$.

The geometrical meaning of $\left(\mathrm{HS}^{\Sigma}\right)$ is illustrated on Figure 3 .

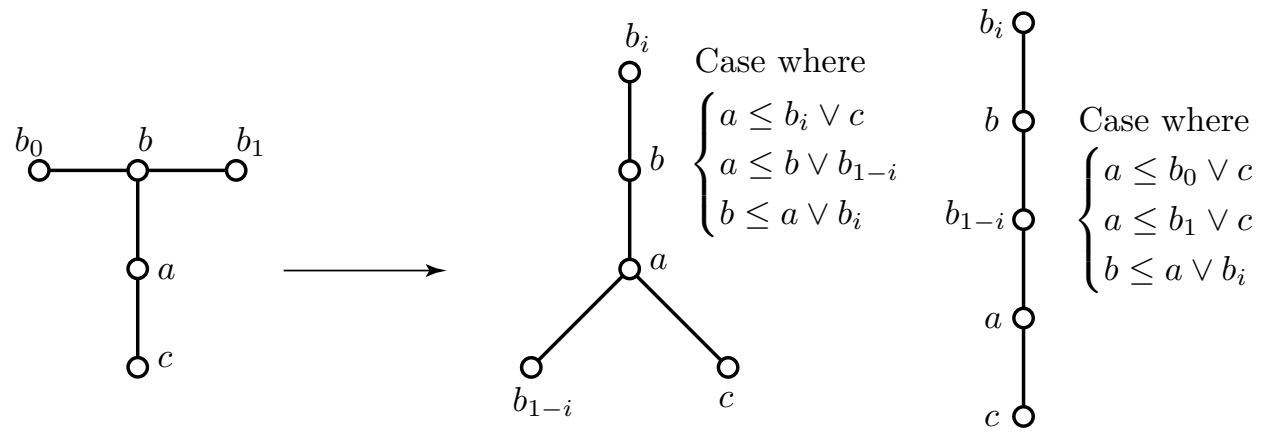

FiguRE 3. Illustrating $\left(\mathrm{HS}^{\Sigma}\right)$

Lemma 5.4. Let $L$ be a lattice, let $\Sigma$ be a subset of $\mathrm{J}(L)$. Then the following statements hold:

(i) If $L$ satisfies (HS), then $L$ satisfies $\left(\mathrm{HS}^{\Sigma}\right)$.

(ii) If $\Sigma$ is a join-seed of $L$ and $L$ satisfies $\left(\mathrm{HS}^{\Sigma}\right)$, then $L$ satisfies (HS).

Proof. (i) Let $a, b, c, b_{0}, b_{1} \in \Sigma$ satisfy the premise of $\left(\mathrm{HS}^{\Sigma}\right)$. Observe that $b^{\prime}=b \wedge\left(b_{0} \vee b_{1}\right)=b$ and $a \wedge\left(b^{\prime} \vee c\right)=a$. Since $a \leq b \vee c$ is minimal in $b$ and $b \wedge b_{i}<b$, it follows from the join-irreducibility of $a$ that there exists $i<2$ such that one of the following inequalities holds:

$$
\begin{aligned}
& a \leq\left(\left(b \wedge\left(a \vee b_{i}\right)\right) \vee c\right) \wedge\left(b_{i} \vee c\right) \wedge\left(b \vee b_{1-i}\right), \\
& a \leq\left(\left(b \wedge\left(a \vee b_{i}\right)\right) \vee c\right) \wedge\left(b_{0} \vee c\right) \wedge\left(b_{1} \vee c\right) .
\end{aligned}
$$

From the minimality of $b$ in $a \leq b \vee c$ it follows that $b \leq a \vee b_{i}$. Furthermore, in the first case $a \leq b_{i} \vee c, b \vee b_{1-i}$ while in the second case $a \leq b_{0} \vee c, b_{1} \vee c$.

(ii) Let $d$ (resp., $e$ ) denote the left hand side (resp., right hand side) of the identity (HS). Let $p \in \Sigma$ such that $p \leq d$, we prove that $p \leq e$. If $p \leq b^{\prime}$ then $p \leq d \wedge b^{\prime}=a \wedge b^{\prime}$, if $p \leq c$ then $p \leq a \wedge c$, in both cases $p \leq e$. Suppose from now on that $p \not \leq b^{\prime}, c$. Since $\Sigma$ is a join-seed of $L$, there are $u \leq b^{\prime}$ and $v \leq c$ in $\Sigma$ such 
that $p \leq u \vee v$ is a minimal nontrivial join-cover. If $u \leq b_{i}$, for some $i<2$, then $u \leq b \wedge b_{i}$, whence

$$
p \leq a \wedge(u \vee v) \leq a \wedge\left(\left(b \wedge b_{i}\right) \vee c\right) \leq e .
$$

Suppose from now on that $u \not \leq b_{0}, b_{1}$. Since $\Sigma$ is a join-seed of $L$, there are $u_{0} \leq b_{0}$ and $u_{1} \leq b_{1}$ in $\Sigma$ such that $u \leq u_{0} \vee u_{1}$ is a minimal nontrivial join-cover. By $\left(\mathrm{HS}^{\Sigma}\right)$, there exists $i<2$ such that $u \leq p \vee u_{i}$ and either $p \leq u_{i} \vee v, u \vee u_{1-i}$ or $p \leq u_{0} \vee v, u_{1} \vee v$. In the first case,

$$
p \leq a \wedge\left(u_{i} \vee v\right) \wedge\left(u \vee u_{1-i}\right) \wedge\left(\left(u \wedge\left(p \vee u_{i}\right)\right) \vee v\right) \leq e .
$$

In the second case,

$$
p \leq a \wedge\left(u_{0} \vee v\right) \wedge\left(u_{1} \vee v\right) \wedge\left(\left(u \wedge\left(p \vee u_{i}\right)\right) \vee v\right) \leq e
$$

Since every element of $L$ is a join of elements of $\Sigma$, we obtain that $d \leq e$. Since $e \leq d$ holds in any lattice, we obtain that $d=e$.

Corollary 5.5. The lattice $\mathbf{C o}(T)$ satisfies (HS), for every chain $(T, \unlhd)$.

Proof. We apply Lemma 5.4 to $L=\mathbf{C o}(T)$ together with the join-seed $\Sigma=$ $\{\{p\} \mid p \in T\}$. Let $a, b, c, b_{0}, b_{1} \in T$ such that $a \neq b$, the inequality $\{a\} \leq\{b\} \vee\{c\}$ is minimal in $b$ (thus $a \neq c$ ), and $\{b\} \leq\left\{b_{0}\right\} \vee\left\{b_{1}\right\}$. Since $\mathbf{C o}(T, \unlhd)=\mathbf{C o}(T, \unrhd)$, we may assume without loss of generality that $c \triangleleft a \triangleleft b$. Furthermore, there exists $i<2$ such that $b \unlhd b_{i}$, whence $\{b\} \leq\{a\} \vee\left\{b_{i}\right\}$. Since $T$ is a chain, either $b_{1-i} \unlhd a$ or $a \unlhd b_{1-i}$. In the first case, $\{a\} \leq\left\{b_{i}\right\} \vee\{c\},\{b\} \vee\left\{b_{1-i}\right\}$. In the second case, $\{a\} \leq\left\{b_{0}\right\} \vee\{c\},\left\{b_{1}\right\} \vee\{c\}$.

Hence $\mathbf{C o}(T)$ satisfies $\left(\mathrm{HS}^{\Sigma}\right)$. By Lemma 5.4 $\mathbf{C o}(T)$ satisfies (HS).

\section{The Transitivity Lemma}

The main purpose of the present section is to prove the following technical lemma, which provides a large supply of minimal coverings.

Lemma 6.1 (The Transitivity Lemma). Let $L$ be a lattice satisfying the identities (HS), (U), (B), (E), and (P), let $\Sigma$ be a join-seed of $L$, and let $a, b, c, b_{0}, b_{1} \in \Sigma$ such that both $a \leq b \vee c$ and $b \leq b_{0} \vee b_{1}$ are minimal nontrivial join-covers. Then there exists $i<2$ such the following statements hold:

(i) the inequality $b \leq a \vee b_{i}$ holds, and both inequalities $b \leq c \vee b_{i}$ and $a \leq c \vee b_{i}$ are minimal nontrivial join-covers;

(ii) one of the following two statements holds:

(ii.1) $a \leq b_{i} \vee c, b_{1-i} \vee b$ and, if $a \neq b_{1-i}$, then the inequality $a \leq b_{0} \vee b_{1}$ is a minimal nontrivial join-cover;

(ii.2) $a \leq b_{0} \vee c, b_{1} \vee c$ and, if $a \neq b_{1-i}$, then the inequality $a \leq b_{1-i} \vee c$ is a minimal nontrivial join-cover.

The situation may be partly viewed on Figure 3 .

Proof. It follows from Lemma 5.4 that there exists $i<2$ such that

$$
b \leq a \vee b_{i} \text { and either } a \leq b_{i} \vee c, b_{1-i} \vee b \text { or } a \leq b_{0} \vee c, b_{1} \vee c .
$$

Since $b \leq b_{i} \vee c$ is a nontrivial join-cover and $\Sigma$ is a join-seed of $L$, there are $x \leq b_{i}$ and $c^{\prime} \leq c$ in $\Sigma$ such that $b \leq x \vee c^{\prime}$ is a minimal nontrivial join-cover. By applying $\left(\mathrm{B}_{\mathrm{j}}\right)$ to the inequalities $b \leq b_{i} \vee b_{1-i}, x \vee c^{\prime}$ and observing that $b \not \leq b_{i}=b_{i} \vee x$, we 
obtain that $b \leq b_{1-i} \vee x$, whence, by the minimality assumption on $b_{i}, x=b_{i}$. By applying Lemma 5.4 to the minimal nontrivial join-covers $a \leq b \vee c$ and $b \leq b_{i} \vee c^{\prime}$, we obtain that either $a \leq c^{\prime} \vee c=c$, a contradiction, or $a \leq b \vee c^{\prime}$. By the minimality assumption on $c$, the latter implies that $c=c^{\prime}$. Hence we have proved the following:

the inequality $b \leq b_{i} \vee c$ is a minimal nontrivial join-cover.

Now we shall proceed by proving the following statement:

the inequality $a \leq b_{i} \vee c$ is a minimal nontrivial join-cover.

If $a \leq b_{i}$, then $b \leq a \vee b_{i}=b_{i}$, a contradiction; whence $a \not \leq b_{i}$. So $a \leq b_{i} \vee c$ is a nontrivial join-cover, thus, since $\Sigma$ is a join-seed of $L$, there are $x \leq b_{i}$ and $c^{\prime} \leq c$ in $\Sigma$ such that $a \leq x \vee c^{\prime}$ is a minimal nontrivial join-cover. By applying $\left(\mathrm{B}_{\mathrm{j}}\right)$ to the inequalities $a \leq b \vee c, x \vee c^{\prime}$ and observing that $a \not \leq c=c \vee c^{\prime}$, we obtain that $a \leq b \vee c^{\prime}$, whence, by the minimality assumption on $c$, we obtain that $c=c^{\prime}$.

Now we apply Lemma 4.2 to the minimal nontrivial join-covers $a \leq b \vee c, x \vee c$ and the inequality $b \leq b_{0} \vee b_{1}$. Thus either $b \leq a \vee x$ or there exists $j<2$ such that $a \leq b_{j} \vee c$ and $b \leq a \vee b_{j}, b_{j} \vee x$. Suppose that the second case holds. If $i \neq j$, then $b \leq a \vee b_{j} \leq c \vee b_{j}$. But $b \leq c \vee b_{i}$ and $b \leq b_{i} \vee b_{j}$, whence, by ( $\left.\mathrm{U}_{\mathrm{j}}\right)$, either $b \leq b_{0}$ or $b \leq b_{1}$ or $b \leq c$, a contradiction. Therefore, $i=j$ and $b \leq x \vee b_{i}=b_{i}$, a contradiction.

Hence the first case holds, thus it follows from $a \leq x \vee c$ that $b \leq x \vee c$ with $x \leq b_{i}$, thus, by (6.2), $x=b_{i}$. This completes the proof of (6.3), and thus also the proof of (i).

Now let us establish the remaining minimal nontrivial join-covers in (ii), under the additional assumption that $a \neq b_{1-i}$. We have already seen that $a \not \leq b_{i}$. If $a \leq b_{1-i}$, then, since $b \leq a \vee b_{i}$ and by the minimality assumption on $b_{1-i}$, we obtain that $a=b_{1-i}$, a contradiction. Therefore, we have obtained the inequalities

$$
a \not \leq b_{0} \text { and } a \not \leq b_{1} \text {. }
$$

Now we separate cases, according to (6.1).

Case 1. $a \leq b_{i} \vee c, b \vee b_{1-i}$. From the second inequality and $b \leq b_{0} \vee b_{1}$ it follows that $a \leq b_{0} \vee b_{1}$. Thus, by (6.4) and since $\Sigma$ is a join-seed of $L$, there are $x_{0} \leq b_{0}$ and $x_{1} \leq b_{1}$ in $\Sigma$ such that $a \leq x_{0} \vee x_{1}$ is a minimal nontrivial join-cover. By applying $\left(\mathrm{B}_{\mathrm{j}}\right)$ to the inequalities $a \leq b_{i} \vee c$ (see (6.3) ) and $a \leq x_{i} \vee x_{1-i}$ and observing that $a\left\lfloor b_{i}=x_{i} \vee b_{i}\right.$, we obtain the inequality $a \leq c \vee x_{i}$ with $x_{i} \leq b_{i}$, thus, by (6.3), $x_{i}=b_{i}$. On the other hand, $b \leq a \vee b_{i} \leq b_{i} \vee x_{1-i}$ with $x_{1-i} \leq b_{1-i}$, thus, by the minimality assumption on $b_{1-i}$, we obtain that $x_{1-i}=b_{1-i}$. Therefore, we have proved the following statement:

the inequality $a \leq b_{0} \vee b_{1}$ is a minimal nontrivial join-cover.

Case 2. $a \leq b_{0} \vee c, b_{1} \vee c$. From (6.4), the inequalities $a \not \leq c$ and $a \leq b_{1-i} \vee c$, and the assumption that $\Sigma$ is a join-seed of $L$, it follows that there are $x \leq b_{1-i}$ and $c^{\prime} \leq c$ in $\Sigma$ such that $a \leq x \vee c^{\prime}$ is a minimal nontrivial join-cover. By applying $\left(\mathrm{B}_{\mathrm{j}}\right)$ to the inequalities $a \leq b \vee c, x \vee c^{\prime}$ and observing that $a \not \leq c=c \vee c^{\prime}$, we obtain that $a \leq b \vee c^{\prime}$, whence, since $c^{\prime} \leq c$ and by the minimality assumption on $c$, we obtain that $c=c^{\prime}$.

Suppose now that $x<b_{1-i}$. Applying Lemma 4.2 to the join covers $a \leq c \vee x, b \vee c$ and $b \leq b_{0} \vee b_{1}$, we obtain that either $b \leq a \vee x$ or $b \leq a \vee b_{j}, x \vee b_{j}$, for some 
$j<2$. In the first case, $b \leq a \vee x \leq c \vee x$. Since $b \not \leq b_{1-i}=b_{1-i} \vee x$, we obtain, by $\left(\mathrm{B}_{\mathrm{j}}\right)$ applied to the inequalities $b \leq b_{0} \vee b_{1}, c \vee x$ that $b \leq b_{i} \vee x$, which contradicts the assumption that the cover $b \leq b_{0} \vee b_{1}$ is a minimal nontrivial join-cover. Hence the second case applies. If $j \neq i$, then $b \leq a \vee b_{j} \leq c \vee b_{j}$, while $b \leq c \vee b_{i}$ and $b \leq b_{i} \vee b_{j}$, whence, by $\left(\mathrm{U}_{\mathrm{j}}\right)$, either $b \leq b_{i}$ or $b \leq b_{j}$ or $b \leq c$, a contradiction. Hence $j=i$ and $b \leq x \vee b_{i}$ with $x<b_{1-i}$, which contradicts the minimality assumption on $b_{1-i}$. This completes the proof of the following statement:

the inequality $a \leq b_{1-i} \vee c$ is a minimal nontrivial join-cover,

and thus the proof of (ii).

In particular, in the context of Lemma 6.2 it follows from (i) that $a D b_{i}$ always holds. Moreover, if $a \neq b_{1-i}$, then, by (ii), $a D b_{1-i}$ holds. Therefore, we obtain the following remarkable corollary.

Corollary 6.2. Let $L$ be a lattice satisfying the identities (HS), (U), (B), (E), and $(\mathrm{P})$, let $\Sigma$ be a join-seed of $L$. For any $a, b, c \in \Sigma$, from $a D b D c$ and $a \neq c$ it follows that a $D c$.

\section{The COnstruction}

In this section, we shall fix a complete, lower continuous, finitely spatial lattice $L$ satisfying (HS), (U), (B), (E), and (P). By Lemma 5.2 $L$ is dually 2-distributive, thus, by Lemma 2.2 $\Sigma=\mathrm{J}(L)$ is a join-seed of $L$.

For every $a \in \mathrm{J}(L)$, we denote by $\left\{A_{a}, B_{a}\right\}$ the Udav-Bond partition of $[a]^{D}$ associated with $a$, as defined in [12, Section 5]. We define a binary relation $\unlhd_{a}$ on $\mathrm{J}_{a}(L)=\{a\} \cup[a]^{D}$ by the following:

(i) $x \unlhd_{a} a \unlhd_{a} y$ and $x \unlhd_{a} y$, for all $(x, y) \in\left(A_{a} \cup\{a\}\right) \times\left(B_{a} \cup\{a\}\right)$;

(ii) $x \unlhd_{a} y$ iff $y \leq a \vee x$, for all $x, y \in A_{a}$;

(iii) $x \unlhd_{a} y$ iff $x \leq a \vee y$, for all $x, y \in B_{a}$.

We also say that $x \triangleleft_{a} y$ iff $x \unlhd_{a} y$ and $x \neq y$, for all $x, y \in \mathrm{J}_{a}(L)$.

Lemma 7.1. The relation $\unlhd_{a}$ is a total ordering of $\mathrm{J}_{a}(L)$, for any $a \in \mathrm{J}(L)$.

Proof. It is trivial that $\unlhd_{a}$ is reflexive. Let $x, y, z \in \mathrm{J}_{a}(L)$ with $x \unlhd_{a} y$ and $y \unlhd_{a} z$, we prove that $x \unlhd_{a} z$. This is obvious if either $a \in\{x, y, z\}$ or $(x, z) \in A_{a} \times B_{a}$, so suppose otherwise. Then $x$ and $z$ belong to the same block of the Udav-Bond partition associated with $a$, say, $\{x, z\} \subseteq A_{a}$. Since $y \unlhd_{a} z, y$ belongs to $A_{a}$ as well. Furthermore, $z \leq a \vee y \leq a \vee x$ and thus $x \unlhd_{a} z$. The proof for $\{x, z\} \subseteq B_{a}$ is similar. This proves that $\unlhd_{a}$ is transitive.

Let $x, y \in \mathrm{J}_{a}(L)$ such that $x \unlhd_{a} y \unlhd_{a} x$, we prove that $x=y$. This is obvious if $a \in\{x, y\}$, so suppose that $a \notin\{x, y\}$. Then $x$ and $y$ belong to the same block of the Udav-Bond partition associated with $a$, say, $\{x, y\} \subseteq A_{a}$. Pick $u \in B_{a}$. Then $a \vee x=a \vee y$, but $a \leq u \vee x, u \vee y$, thus $u \vee x=u \vee y$, thus, by the join-semidistributivity of $L$ (see Lemma [5.2),

$$
a \leq u \vee x=u \vee y=u \vee(x \wedge y) .
$$

However, by Lemma 2.3 both inequalities $a \leq u \vee x, u \vee y$ are minimal nontrivial join-covers, thus $x=y$. Hence $\unlhd_{a}$ is antisymmetric.

Now let $x, y \in \mathrm{J}_{a}(L)$, we prove that either $x \unlhd_{a} y$ or $y \unlhd_{a} x$. This is obvious if either $a \in\{x, y\}$ or $x$ and $y$ belong to different blocks of the Udav-Bond partition associated with $a$, so suppose otherwise, say, $\{x, y\} \subseteq B_{a}$. Pick $u \in A_{a}$. By 
Lemma 2.3 both inequalities $a \leq u \vee x, u \vee y$ are minimal nontrivial join-covers, thus, by applying Lemma 3.2 to the minimal nontrivial join-covers $a \leq u \vee x, u \vee y, u \vee y$, we obtain that either $x \leq a \vee y$ or $y \leq a \vee x$, thus either $x \unlhd_{a} y$ or $y \unlhd_{a} x$. The proof for $\{x, y\} \subseteq A_{a}$ is similar. Hence $\unlhd_{a}$ is a total ordering.

For any $a \in \mathrm{J}(L)$, let $\varphi_{a}: L \rightarrow \mathcal{P}\left(\mathrm{J}_{a}(L)\right)$ be the map defined by the rule

$$
\varphi_{a}(x)=\left\{b \in \mathrm{J}_{a}(L) \mid b \leq x\right\}, \text { for all } x \in L .
$$

Lemma 7.2. The set $\varphi_{a}(x)$ is order-convex in $\left(\mathrm{J}_{a}(L), \unlhd_{a}\right)$, for any $x \in L$.

Proof. Let $u, v, w \in \mathrm{J}_{a}(L)$ such that $u \triangleleft_{a} w \triangleleft_{a} v$ and $u, v \leq x$, we prove that $w \leq x$. If $u \in\{a\} \cup A_{a}$ and $v \in\{a\} \cup B_{a}$, then $a \leq u \vee v \leq x$, and then, $w \in\{a\} \cup A_{a}$ implies that $w \leq a \vee u \leq x$, while $w \in\{a\} \cup B_{a}$ implies that $w \leq a \vee v \leq x$.

Suppose now that $u, v \in A_{a}$. From $w \unlhd_{a} v$ it follows that $w \in A_{a}$. Pick $t \in B_{a}$. By Lemma 2.3. all inequalities $a \leq t \vee u, t \vee v, t \vee w$ are minimal nontrivial joincovers; from $a \vee v \leq a \vee w \leq a \vee u$ it follows that $t \vee v \leq t \vee w \leq t \vee u$, thus, by Lemma 3.4 $w \leq u \vee v \leq x$. The argument is similar in case $u, v \in B_{a}$.

Lemma 7.3. The map $\varphi_{a}$ is a lattice homomorphism from $L$ to $\mathbf{C o}\left(\mathrm{J}_{a}(L)\right)$, and it preserves the existing bounds.

Proof. It is clear that $\varphi_{a}$ is a meet-homomorphism from $L$ to $\mathbf{C o}\left(\mathrm{J}_{a}(L)\right)$ and that it preserves the existing bounds. Let $x, y \in L$, we prove that $\varphi_{a}(x \vee y)=\varphi_{a}(x) \vee \varphi_{a}(y)$. It suffices to prove that $b \in \varphi_{a}(x \vee y)$ implies that $b \in \varphi_{a}(x) \vee \varphi_{a}(y)$, for any $b \in \mathrm{J}_{a}(L)$. This is trivial if $b \in \varphi_{a}(x) \cup \varphi_{a}(y)$, so suppose otherwise, that is, $b \not \leq x, y$. Since $b \leq x \vee y$ and $\mathrm{J}(L)$ is a join-seed of $L$, there are $b_{0} \leq x$ and $b_{1} \leq y$ in $\mathrm{J}(L)$ such that the inequality $b \leq b_{0} \vee b_{1}$ is a minimal nontrivial join-cover. From Corollary 6.2 it follows that both $b_{0}$ and $b_{1}$ belong to $\mathrm{J}_{a}(L)$. If $b=a$, then the pair $\left(b_{0}, b_{1}\right)$ belongs either to $A_{a} \times B_{a}$ or $B_{a} \times A_{a}$. In the first case, $b_{0} \unlhd_{a} a \unlhd_{a} b_{1}$, in the second case, $b_{1} \unlhd_{a} a \unlhd_{a} b_{0}$; in both cases, $b=a \in \varphi_{a}(x) \vee \varphi_{a}(y)$.

Suppose from now on that $b \neq a$, say, $b \in B_{a}$. Pick $c \in J(L)$ such that $a \leq b \vee c$ is a minimal nontrivial join-cover; observe that $c \in A_{a}$. So there exists $i<2$ such that the statements (i), (ii) of Lemma 6.1 hold.

From the fact that the inequality $a \leq b_{i} \vee c$ is a minimal nontrivial join-cover and $c \in A_{a}$ it follows that $b_{i} \in B_{a}$. From the relations $b, b_{i} \in B_{a}, b \neq b_{i}$, and $b \leq a \vee b_{i}$ it follows that

$$
b \triangleleft_{a} b_{i} .
$$

If $a=b_{1-i}$, then, since $b \in B_{a}$, we obtain that $b_{1-i}=a \triangleleft_{a} b$, thus, by (7.1), $b \in \varphi_{a}(x) \vee \varphi_{a}(y)$. Suppose from now on that $a \neq b_{1-i}$. If (ii.1) of Lemma 6.1 holds, then the inequality $a \leq b_{0} \vee b_{1}$ is a minimal nontrivial join-cover with $b_{i} \in B_{a}$, thus $b_{1-i} \in A_{a}$, thus $b_{1-i} \triangleleft_{a} b$, which, together with (7.1), implies that $b \in \varphi_{a}(x) \vee \varphi_{a}(y)$. Suppose now that (ii.2) of Lemma 6.1 holds. From the fact that $a \leq b_{1-i} \vee c$ is a minimal nontrivial join-cover and $c \in A_{a}$ it follows that $b_{1-i} \in B_{a}$. If $b \unlhd_{a} b_{1-i}$, then, since $b, b_{1-i} \in B_{a}$, we obtain that $b \leq a \vee b_{1-i} \leq c \vee b_{1-i}$, but $b \leq c \vee b_{i}$ and $b \leq b_{i} \vee b_{1-i}$, whence, by $\left(\mathrm{U}_{\mathrm{j}}\right)$, either $b \leq b_{0}$ or $b \leq b_{1}$ or $b \leq c$, a contradiction. Hence $b \unlhd_{a} b_{1-i}$, thus, by Lemma 7.1] $b_{1-i} \unlhd_{a} b$. Therefore, it follows again from (7.1) that $b \in \varphi_{a}(x) \vee \varphi_{a}(y)$. 


\section{THE REPRESENTATION THEOREM}

Notation 8.1. Let $\mathbf{S U B}(\mathcal{L O})$ denote the class of all lattices that can be embedded into a direct product of the form $\prod_{i \in I} \mathbf{C o}\left(T_{i}\right)$, where $\left\langle T_{i} \mid i \in I\right\rangle$ is a family of chains.

Our main theorem is the following.

Theorem 8.2. For a lattice $L$, the following are equivalent:

(i) $L$ belongs to $\mathbf{S U B}(\mathcal{L O})$.

(ii) L satisfies the identities (HS), (U), (B), (E), and (P).

(iii) There exists an embedding $\varphi: L \hookrightarrow \prod_{i \in I} \mathbf{C o}\left(T_{i}\right)$, for some family $\left\langle T_{i} \mid i \in I\right\rangle$ of chains, which preserves the existing bounds and satisfies the following additional properties:

- if $L$ is finite, then $\sum_{i \in I}\left|T_{i}\right| \leq|\mathrm{J}(L)|^{2}$;

- if $L$ is subdirectly irreducible, then $I=\{0\}, \varphi$ is atom-preserving, and, if $L$ is finite, then $\left|T_{0}\right|=|\mathrm{J}(L)|$.

- if $L$ is finite, atomistic, and subdirectly irreducible, then $L \cong \mathbf{C o}(n)$, where $n=|\mathrm{J}(L)|$.

Proof. (i) $\Rightarrow$ (ii) We have seen in [12 that $L$ satisfies (U) and (B). Moreover, it follows from Corollaries 3.3 4.3 and [5.5 that $L$ satisfies (E), (P), and (HS).

(ii) $\Rightarrow$ (iii) As in 12, 13, we embed $L$ into the filter lattice $\widehat{L}$ of $L$, partially ordered by reverse inclusion. This embedding preserves the existing bounds and atoms. We recall that $\widehat{L}$ is complete, lower continuous, and finitely spatial. Let $\mathrm{J}_{a}(\widehat{L})$ and $\varphi_{a}: \widehat{L} \rightarrow \mathbf{C o}\left(\mathrm{J}_{a}(\widehat{L})\right)$ be defined as in Section 7 and let $\psi_{a}: L \rightarrow \mathbf{C o}\left(\mathrm{J}_{a}(\widehat{L})\right)$ be the restriction of $\varphi_{a}$ to $L$, for any $a \in \mathrm{J}(\widehat{L})$. Since every element of $\widehat{L}$ is a join of elements of $\mathrm{J}(\widehat{L})$, it follows from Lemma 7.3 that the map $\psi: L \rightarrow \prod_{a \in \mathrm{J}(\widehat{L})} \mathbf{C o}\left(\mathrm{J}_{a}(\widehat{L})\right)$ that with any $x \in L$ associates the family $\left\langle\psi_{a}(x) \mid a \in \mathrm{J}(\widehat{L})\right\rangle$ is a lattice embedding; it obviously preserves the existing bounds. In case $L$ is finite, we have $\widehat{L}=L$ and $\left|\mathrm{J}_{a}(L)\right| \leq|\mathrm{J}(L)|$, for all $a \in \mathrm{J}(L)$; the cardinality bound follows immediately.

Suppose now that $L$ is subdirectly irreducible. Thus $\psi_{a}$ is an embedding, for some $a \in \mathrm{J}(\widehat{L})$; pick such an $a$. Every atom $x$ of $L$ is also an atom of $\widehat{L}$, and $\psi_{a}(x)$ is nonempty, thus there exists $b \in \mathrm{J}_{a}(\widehat{L})$ below $x$, whence $x=b \in \mathrm{J}_{a}(\widehat{L})$ and $\psi_{a}(x)=\{x\}$, an atom of $\mathbf{C o}\left(\mathrm{J}_{a}(L)\right)$. Suppose now that $L$ is finite, thus $\widehat{L}=L$. For any $x \in \mathrm{J}(L)$, if $x^{\prime}$ denotes the join of all elements of $\mathrm{J}_{a}(L)$ below $x$, then $\psi_{a}(x)=\psi_{a}\left(x^{\prime}\right)$, whence $x=x^{\prime}$, thus, since $x$ is join-irreducible, $x \in \mathrm{J}_{a}(L)$; therefore, $\mathrm{J}_{a}(L)=\mathrm{J}(L)$.

Now suppose, in addition, that $L$ is atomistic. Then $\{x\}=\psi_{a}(x)$ belongs to the range of $\psi_{a}$, for any $x \in \mathrm{J}(L)$, thus $\psi_{a}$ is surjective, hence it is an isomorphism from $L$ onto $\mathbf{C o}\left(\mathrm{J}(L), \unlhd_{a}\right)$.

(iii) $\Rightarrow$ (i) is trivial.

Remark 8.3. A finite, atomistic lattice $L$ in $\mathbf{S U B}(\mathcal{L O})$ may not embed atompreservingly into any $\mathbf{C o}(P)$, thus a fortiori into any product of the form $\prod_{i \in I} \mathbf{C o}\left(T_{i}\right)$ where the $T_{i}$-s are chains, as shows [12, Example 8.1]. Also, a finite, atomistic, subdirectly irreducible lattice in SUB may not be of the form $\mathbf{C o}(P)$, see [12, Example 8.2].

Corollary 8.4. The class $\mathbf{S U B}(\mathcal{L O})$ is a finitely based variety of lattices. In particular, $\mathbf{S U B}(\mathcal{L O})$ is closed under homomorphic images. 
This result solves positively Problem 3 in 12 .

\section{The Class $\mathbf{S U B}(n)$, FOR $n \geq 0$}

We start with the following lemma.

Lemma 9.1. Let $L$ be a complete, lower continuous, finitely spatial lattice in $\operatorname{SUB}(\mathcal{L O})$, let $a \in \mathrm{J}(L)$. Let $x, y, u \in[a]^{D}$ such that $a \leq u \vee x, u \vee y$. If $x \leq a \vee y$, then the inequality $x \leq u \vee y$ is a minimal nontrivial join-cover.

Proof. From Lemma 2.3 it follows that both inequalities $a \leq u \vee x, u \vee y$ are minimal nontrivial join-covers. Since $x \leq a \vee y$ and $a \leq u \vee y$, we obtain that $x \leq u \vee y$. From Lemma 2.4 it follows that $x \not \leq u, y$. Since $x \leq u \vee y$ and $\mathrm{J}(L)$ is a join-seed of $L$, there are $u^{\prime} \leq u$ and $y^{\prime} \leq y$ in $\mathrm{J}(L)$ such that the inequality $x \leq u^{\prime} \vee y^{\prime}$ is a minimal nontrivial join-cover. So $a \leq x \vee u \leq y^{\prime} \vee u$ with $y^{\prime} \leq y$, thus, by the minimality of $y$ in $a \leq y \vee u$, we obtain that $y^{\prime}=y$. If $u^{\prime}=a$, then $a \leq u$, a contradiction; whence $u^{\prime} \neq a$; but $a D x D u^{\prime}$, whence, by Corollary6.2 $a D u^{\prime}$. But $u^{\prime} \leq u$ and $a D u$, whence, by Lemma $2.4 u^{\prime}=u$.

Now we are able to relate chains in the $\mathrm{J}_{a}(L)$-s and Stirlitz tracks.

Corollary 9.2. Let $L$ be a complete, lower continuous, finitely spatial lattice in $\operatorname{SUB}(\mathcal{L O})$, let $a \in \mathrm{J}(L)$, let $n$ be a natural number, let $u, x_{0}, \ldots, x_{n} \in \mathrm{J}_{a}(L)$ with $x_{0} \triangleleft_{a} x_{1} \triangleleft_{a} \cdots \triangleleft_{a} x_{n}$. Denote by $\left\{A_{a}, B_{a}\right\}$ the Udav-Bond partition of $[a]^{D}$ associated with a. Then the following statements hold:

(i) If $u \in A_{a}$ and $x_{0}, \ldots, x_{n} \in\{a\} \cup B_{a}$, then $\left(\left\langle x_{i} \mid 0 \leq i \leq n\right\rangle,\langle u \mid 1 \leq i \leq n\rangle\right)$ is a Stirlitz track.

(ii) If $u \in B_{a}$ and $x_{0}, \ldots, x_{n} \in\{a\} \cup A_{a}$, then $\left(\left\langle x_{n-i} \mid 0 \leq i \leq n\right\rangle,\langle u \mid 1 \leq i \leq n\rangle\right)$ is a Stirlitz track.

Proof. (i) It follows from Lemma 9.1 that the inequality $x_{i} \leq u \vee x_{i+1}$ is a minimal nontrivial join-cover, for any $i \in\{0, \ldots, n-1\}$; the conclusion follows. The proof for (ii) is similar.

We recall, see [13, that for any positive integer $n$, the class $\mathbf{S U B}_{n}$ of all lattices that can be embedded into some $\mathbf{C o}(P)$ where $P$ is a poset of length at most $n$ is a finitely based variety, defined by the identities $(\mathrm{S}),(\mathrm{U}),(\mathrm{B})$, together with new identities $\left(\mathrm{H}_{n}\right)$ and $\left(\mathrm{H}_{k, n+1-k}\right)$ for $1 \leq k \leq n$.

Notation 9.3. For a natural number $n$, let $\mathbf{S U B}(n)$ denote the class of all lattices that can be embedded into a power of $\mathbf{C o}(n)$.

Of course, $\mathbf{S U B}(0)$ is the trivial variety while $\mathbf{S U B}(1)=\mathbf{S U B}(2)$ is the class of all distributive lattices. Now we obtain the main result of this section.

Theorem 9.4. Let $n$ be a positive integer. The class $\mathbf{S U B}(n+1)$ is a finitely generated variety, defined by the identities (HS), (U), (B), (E), (P), and $\left(\mathrm{H}_{k, n+1-k}\right)$ for $1 \leq k \leq n$. Furthermore, $\mathbf{S U B}(n+1)=\mathbf{S U B}(\mathcal{L O}) \cap \mathbf{S U B}_{n}$.

Proof. Since the $(n+1)$-element chain belongs to $\mathbf{S U B}_{n}$, the containment $\mathbf{S U B}(n+1) \subseteq \mathbf{S U B}(\mathcal{L} \mathcal{O}) \cap \mathbf{S U B}_{n}$ is obvious. Furthermore, by the results of [13] and Theorem 8.2 every lattice in $\mathbf{S U B}(\mathcal{L O}) \cap \mathbf{S U B}_{n}$ satisfies the identities (HS), $(\mathrm{U}),(\mathrm{B}),(\mathrm{E}),(\mathrm{P})$, and $\left(\mathrm{H}_{k, n+1-k}\right)$ for $1 \leq k \leq n$.

Now let $L$ be a lattice satisfying the identities (HS), (U), (B), (E), (P), and $\left(\mathrm{H}_{k, n+1-k}\right)$ for $1 \leq k \leq n$, we prove that $L$ belongs to $\mathbf{S U B}(n+1)$. By embedding $L$ 
into its filter lattice, we see that it suffices to consider the case where $L$ is complete, lower continuous, and finitely spatial. By Theorem 8.2 $L$ belongs to $\mathbf{S U B}(\mathcal{L O})$. In order to conclude the proof, it suffices to establish that $\mathrm{J}_{a}(L)$ has at most $n+1$ elements, for any $a \in \mathrm{J}(L)$. If this is not the case, then both blocks $A_{a}$ and $B_{a}$ of the Udav-Bond partition of $[a]^{D}$ associated with $a$ are nonempty, and $\mathrm{J}_{a}(L)$ has a chain of the form

$$
x_{k} \triangleleft_{a} \cdots \triangleleft_{a} x_{1} \triangleleft_{a} x_{0}=a=y_{0} \triangleleft_{a} y_{1} \triangleleft_{a} \cdots \triangleleft_{a} y_{l},
$$

where $k$ and $l$ are positive integers with $k+l=n+1$. Define pairs $\sigma$ and $\tau$ by

$$
\begin{aligned}
\sigma & =\left(\left\langle x_{i} \mid 0 \leq i \leq k\right\rangle,\left\langle y_{1} \mid 1 \leq i \leq k\right\rangle\right), \\
\tau & =\left(\left\langle y_{j} \mid 0 \leq j \leq l\right\rangle,\left\langle x_{1} \mid 1 \leq j \leq l\right\rangle\right) .
\end{aligned}
$$

It follows from Corollary 9.2 that both $\sigma$ and $\tau$ are Stirlitz tracks, but $a \leq x_{1} \vee$ $y_{1}$, thus the pair $(\sigma, \tau)$ is a bi-Stirlitz track (see [13]) of index $(k, l)$ with $k+$ $l=n+1$, which contradicts the fact that $L$ satisfies the identity $\left(\mathrm{H}_{k, l}\right)$, see 13 , Proposition 6.2].

In particular, we have proved that $\mathbf{S U B}(n+1)$ is a variety. Of course, it is generated by the single finite lattice $\mathbf{C o}(n+1)$.

Since the construction underlying Theorem 9.4 is the same as the one underlying Theorem 8.2 the corresponding additional information is preserved. For example, any member $L$ of $\mathbf{S U B}(n+1)$ has an embedding into a power of $\mathbf{C o}(n+1)$ which preserves the zero if it exists; furthermore, if $L$ is subdirectly irreducible, then this embedding preserves atoms.

Theorem 9.5. The variety $\mathbf{S U B}(\mathcal{L O})$ is locally finite.

Proof. For a lattice $L$, let $\mathbf{C s u b}(L)$ denote the lattice of all convex sublattices of $L$, ordered by inclusion. For a variety $\mathbf{V}$ of lattices, let $\mathbf{C s u b}(\mathbf{V})$ denote the variety generated by all lattices of the form $\mathbf{C s u b}(L)$, for $L \in \mathbf{V}$. For a chain $T$, the equality $\mathbf{C o}(T)=\mathbf{C s u b}(T)$ obviously holds, whence $\mathbf{S U B}(\mathcal{L} \mathcal{O})$ is a subvariety of $\mathbf{C s u b}(\mathbf{D})$, where $\mathbf{D}$ denotes the variety of all distributive lattices. It is proved in V. Slavík [14] that $\operatorname{Csub}(\mathbf{D})$ is locally finite, therefore, the smaller variety $\mathbf{S U B}(\mathcal{L O})$ is also locally finite.

Corollary 9.6. The variety $\mathbf{S U B}(\mathcal{L O})$ is generated by $\mathbf{C o}(\omega)$, where $\omega$ denotes the chain of natural numbers.

If, for a poset $P$, we denote by $\mathbf{S U B}(P)$ the variety generated by $\mathbf{C o}(P)$, we obtain the 'equation' $\mathbf{S U B}(\mathcal{L O})=\mathbf{S U B}(\omega)$.

Corollary 9.7. The variety $\mathbf{S U B}(\mathcal{L O})$ is the quasivariety join of all varieties $\operatorname{SUB}(n)$, where $1 \leq n<\omega$.

Proof. Let $\mathbf{Q}$ be any quasivariety containing $\mathbf{S U B}(n)$, for every positive integer $n$. Every finite lattice $L$ in $\mathbf{S U B}(\mathcal{L O})$ embeds into a finite power of some $\mathbf{C o}(n)$, thus it belongs to $\mathbf{Q}$. By Theorem 0.5 it follows that $\mathbf{Q}$ contains $\mathbf{S U B}(\mathcal{L O})$.

\section{WEAK StiRlitz TRACKS IN LATTICES OF CONVEX SUBSETS OF CHAins}

Definition 10.1. Let $L$ be a lattice, let $m, n$ be positive integers. 
(i) A weak Stirlitz track of length $n$ of $L$ is a pair $\sigma=\left(\left\langle x_{i} \mid 0 \leq i \leq n\right\rangle, x\right)$, where $x, x_{i}$ (for $0 \leq i \leq n$ ) are elements of $L$, and the following relations hold:

(1) $x_{0} \neq\left(x_{0} \wedge x_{1}\right) \vee\left(x_{0} \wedge x\right)$

(2) $x_{k} \leq x_{k+1} \vee x$, for all $k \in\{0, \ldots, n-1\}$;

(3) $x_{k-1} \not \leq\left(x_{k} \wedge x_{k+1}\right) \vee x$, for all $k \in\{1, \ldots, n-1\}$.

(ii) A weak bi-Stirlitz track of index $(m, n)$ of $L$ is a pair $(\sigma, \tau)$, where $\sigma=$ $\left(\left\langle x_{i} \mid 0 \leq i \leq m\right\rangle, x\right)$ and $\tau=\left(\left\langle y_{j} \mid 0 \leq j \leq n\right\rangle, y\right)$ are both weak Stirlitz tracks such that $x_{0}=y_{0} \leq x_{1} \vee y_{1}$ while $x_{0} \neq\left(x_{0} \wedge x_{1}\right) \vee\left(x_{0} \wedge y_{1}\right)$.

For a Stirlitz track $\sigma=\left(\left\langle x_{i} \mid \quad 0 \leq i \leq n\right\rangle,\left\langle x_{i}^{\prime} \mid \quad 1 \leq i \leq n\right\rangle\right)$, we put $\bar{\sigma}=\left(\left\langle x_{i} \mid 0 \leq i \leq n\right\rangle, x_{1}^{\prime}\right)$, and $\tilde{\sigma}=\left\langle x_{i} \mid 0 \leq i \leq n\right\rangle$, the trace of $\sigma$ and of $\bar{\sigma}$. The trace of a (weak) bi-Stirlitz track $(\sigma, \tau)$ is the pair $(\tilde{\sigma}, \tilde{\tau})$.

Lemma 10.2. Let $L$ be a lattice in SUB. Then the following statements hold:

(i) The pair $\bar{\sigma}$ is a weak Stirlitz track of $L$, for every Stirlitz track $\sigma$ of $L$.

(ii) The pair $(\bar{\sigma}, \bar{\tau})$ is a weak bi-Stirlitz track of L, for every bi-Stirlitz track $(\sigma, \tau)$ of $L$.

Proof. (i) Let $\sigma=\left(\left\langle x_{i} \mid 0 \leq i \leq n\right\rangle,\left\langle x_{i}^{\prime} \mid 1 \leq i \leq n\right\rangle\right)$ be a Stirlitz track of $L$. We put $x=x_{1}^{\prime}$ and we verify (1)-(3) of Definition 10.1(i).

The inequality (1) is trivial, while the inequality (2) follows from [12, Lemma 5.6]. Suppose that $x_{k-1} \leq\left(x_{k} \wedge x_{k+1}\right) \vee x_{1}^{\prime}$. If $x_{k-1} \leq x_{1}^{\prime}$, then, again by [12, Lemma 5.6], $x_{0} \leq x_{k-1} \vee x_{1}^{\prime}=x_{1}^{\prime}$, a contradiction; whence $x_{k-1} \not \leq x_{1}^{\prime}$. Furthermore, $x_{k-1} \not \leq x_{k}$, thus $x_{k-1} \not \leq x_{k} \wedge x_{k+1}$. By applying (B) to the inequalities $x_{k-1} \leq x_{k} \vee x_{k}^{\prime},\left(x_{k} \wedge\right.$ $\left.x_{k+1}\right) \vee x_{1}^{\prime}$ and observing that $x_{k-1} \not \leq x_{k}=x_{k} \vee\left(x_{k} \wedge x_{k+1}\right)$, we obtain that $x_{k-1} \leq\left(x_{k} \wedge x_{k+1}\right) \vee x_{k}^{\prime}$ with $x_{k} \wedge x_{k+1}<x_{k}$, which contradicts the minimality assumption on $x_{k}$.

(ii) follows immediately from (i) and the join-irreducibility of $x_{0}$.

For subsets $X$ and $Y$ of a chain $(T, \unlhd)$, let $X \triangleleft Y$ and $X \unlhd_{\mathrm{w}} Y$ be the following statements:

$$
\begin{aligned}
X \triangleleft Y & \rightleftharpoons x \triangleleft y, \text { for all }(x, y) \in X \times Y, \\
X \unlhd_{\mathrm{w}} Y & \rightleftharpoons \forall x \in X, \exists y \in Y \text { such that } x \unlhd y .
\end{aligned}
$$

Of course, the equivalence

$$
X \triangleleft Y \Longleftrightarrow\left(X \unlhd_{\mathrm{w}} Y \text { and } X \cap Y=\varnothing\right)
$$

holds, for all nonempty $X, Y \in \mathbf{C o}(T)$.

Lemma 10.3. Let $(T, \unlhd)$ be a chain, let $L$ be a sublattice of $\mathbf{C o}(T)$, let $n$ be a positive integer. For any weak Stirlitz track $\left(\left\langle X_{i} \mid 0 \leq i \leq n\right\rangle, X\right)$ of $L$, either the following statement or its dual holds:

$$
X \unlhd_{\mathrm{w}} X_{0} \unlhd_{\mathrm{w}} X_{1} \text { and } X \triangleleft X_{1} \triangleleft X_{2} \triangleleft \cdots \triangleleft X_{n} .
$$

Proof. If $X_{1} \cap X \neq \varnothing$, then $Y \cap\left(X_{1} \vee X\right)=\left(Y \cap X_{1}\right) \vee(Y \cap X)$ for any $Y \in \mathbf{C o}(T)$, a contradiction for $Y=X_{0}$. Hence $X_{1} \cap X=\varnothing$. It follows that either $X \triangleleft X_{1}$ or $X_{1} \triangleleft X$, say, $X \triangleleft X_{1}$. Since $X_{0} \leq X_{1} \vee X$ is a nontrivial join-cover, we obtain that $X \unlhd_{\mathrm{w}} X_{0} \unlhd_{\mathrm{w}} X_{1}$.

Now we prove, by induction on $k$, that the statement

$$
X \triangleleft X_{1} \triangleleft X_{2} \triangleleft \cdots \triangleleft X_{k}
$$


holds, for any $k \in\{1, \ldots, n\}$. For $k=1$ this is already verified. Suppose having established (10.1) at step $k$, with $1 \leq k<n$.

Suppose that $X_{k} \nexists_{\mathrm{w}} X_{k+1}$, that is, there exists $x \in X_{k}$ such that $y \triangleleft x$ holds for any $y \in X_{k+1}$. By the induction hypothesis, this also holds for any $y \in X$, thus $x \notin X \vee X_{k+1}$, which contradicts the assumption that $X_{k} \subseteq X \vee X_{k+1}$. Hence we have proved the relation

$$
X_{k} \unlhd_{\mathrm{w}} X_{k+1} .
$$

Suppose that $X_{k} \cap X_{k+1} \neq \varnothing$. Since $X \unlhd_{\mathrm{w}} X_{k-1} \unlhd_{\mathrm{w}} X_{k} \unlhd_{\mathrm{w}} X_{k+1}$, we obtain that $X_{k-1} \subseteq\left(X_{k} \cap X_{k+1}\right) \vee X$, a contradiction. Hence we have established the relation

$$
X_{k} \cap X_{k+1}=\varnothing .
$$

From (10.2) and (10.3) it follows that $X_{k} \triangleleft X_{k+1}$, which completes the induction step for (10.1). For $k=n$, we obtain the conclusion of the lemma.

Lemma 10.4. Let $(T, \unlhd)$ be a chain, let $L$ be a sublattice of $\mathbf{C o}(T)$, let $m$ and $n$ be positive integers, let $(\sigma, \tau)$ be a weak bi-Stirlitz track of $\mathbf{C o}(T)$ of index $(m, n)$, with

$$
\begin{aligned}
& \sigma=\left(\left\langle X_{i} \mid 0 \leq i \leq m\right\rangle, X\right), \\
& \tau=\left(\left\langle Y_{j} \mid 0 \leq j \leq n\right\rangle, Y\right) .
\end{aligned}
$$

Then $X_{1} \vee Y_{1} \neq X_{1} \cup Y_{1}$, and, putting $Z=X_{0}=Y_{0}$, either the following statement or its dual holds:

$$
X_{m} \triangleleft \cdots \triangleleft X_{1} \triangleleft Y_{1} \triangleleft \cdots \triangleleft Y_{n} \text { and } X_{1} \unlhd_{\mathrm{w}} Z \unlhd_{\mathrm{w}} Y_{1} .
$$

Furthermore, $Z$ does not meet simultaneously $X_{1}$ and $Y_{1}$, and $\mathbf{C o}(m+n)$ embeds into $L$.

Proof. It follows from Lemma 10.3 that we may assume, without loss of generality, that the following statement holds:

$$
X_{m} \triangleleft \cdots \triangleleft X_{1} \triangleleft X \text { and } X_{1} \unlhd_{\mathrm{w}} X_{0} \unlhd_{\mathrm{w}} X \text {. }
$$

Suppose that $X_{0} \unlhd_{\mathrm{w}} X_{1}$. Since $X_{1} \unlhd_{\mathrm{w}} X_{0}$ and $X_{0} \nsubseteq X_{1}, X_{1}$ is a proper final segment of $X_{0}$. Thus, from $X_{1} \triangleleft X$ it follows that $X_{0} \triangleleft X$, but $X_{0} \subseteq X_{1} \vee X$, whence $X_{0} \subseteq X_{1}$, a contradiction. Hence we have established the relation

$$
X_{0} \unlhd_{\mathrm{w}} X_{1} \text {. }
$$

Now suppose that $Y_{1} \unlhd_{\mathrm{w}} Y_{0}$ and $Y_{1} \triangleleft Y$. As in the paragraph above, we obtain that $Y_{0} \not \nexists_{\mathrm{w}} Y_{1}$. By (10.5) and since $X_{0}=Y_{0}=Z$, there exists $z \in Z$ such that $y \triangleleft z$ for any $y \in X_{1} \cup Y_{1}$, which contradicts the fact that $Z \subseteq X_{1} \vee Y_{1}$. Therefore, by Lemma 10.3 the following statement holds:

$$
Y \triangleleft Y_{1} \triangleleft \cdots \triangleleft Y_{n} \text { and } Y \unlhd_{\mathrm{w}} Y_{0} \unlhd_{\mathrm{w}} Y_{1} \text {. }
$$

If $X_{1} \vee Y_{1}=X_{1} \cup Y_{1}$, then $Z=\left(Z \cap X_{1}\right) \vee\left(Z \cap Y_{1}\right)$, a contradiction. Hence $X_{1} \vee Y_{1} \neq X_{1} \cup Y_{1}$, in particular, $X_{1} \cap Y_{1}=\varnothing$. This, together with (10.4) and (10.6), establishes the statement

$$
X_{m} \triangleleft \cdots \triangleleft X_{1} \triangleleft Y_{1} \triangleleft \cdots \triangleleft Y_{n} .
$$

Furthermore, if $Z$ meets both $X_{1}$ and $Y_{1}$, then $Z=\left(Z \cap X_{1}\right) \vee\left(Z \cap Y_{1}\right)$, a contradiction.

In particular, sending $\{i\}$ to $X_{m-i}$ for $0 \leq i<m$ and to $Y_{i-m+1}$ for $m \leq i<$ $m+n$ defines a lattice embedding from $\mathbf{C o}(m+n)$ into $L$. 


\section{Subvarieties of $\mathbf{S U B}(\mathcal{L} \mathcal{O})$}

Notation 11.1. For positive integers $m$ and $n$, we set

$$
L_{m, n}=\{X \in \mathbf{C o}(m+n+1) \mid m \in X \Rightarrow m-1 \in X\},
$$

and we put $c_{m}=\{m-1, m\}$. Observe that $c_{m} \in \mathrm{J}\left(L_{m, n}\right)$.

The lattices $L_{m, n}$, for $m+n \leq 4$, are diagrammed on Figure 4 , together with $\mathbf{C o}(3)$ and $\mathbf{C o}(4)$.

Definition 11.2. For positive integers $m$ and $n$, the canonical bi-Stirlitz track of $L_{m, n}$ is defined as $\left(\sigma_{0}, \tau_{0}\right)$, where we put

$$
\begin{aligned}
\sigma_{0} & =\left(\left\langle c_{m},\{m-1\}, \ldots,\{0\}\right\rangle,\langle\{m+n\} \mid 1 \leq i \leq m\rangle\right), \\
\tau_{0} & \left.=\left\langle c_{m},\{m+1\}, \ldots,\{m+n\}\right\rangle,\langle\{0\} \mid 1 \leq j \leq n\rangle\right) .
\end{aligned}
$$

We observe that the relation $\{m-1\}<c_{m}$ (between entries of $\left.\left(\sigma_{0}, \tau_{0}\right)\right)$ holds.

Lemma 11.3. Let $m$ and $n$ be positive integers. Then the following statements hold:

(i) $L_{m, n}$ is a $\langle 0,1\rangle$-sublattice of $\mathbf{C o}(m+n+1)$.

(ii) The join-irreducible elements of $L_{m, n}$ are the singletons $\{i\}$, where $0 \leq$ $i \leq m+n$ and $i \neq m$, together with $c_{m}$.

(iii) $L_{m, n}$ is subdirectly irreducible, with monolith (smallest nonzero congruence) $\Theta\left(\{m-1\}, c_{m}\right)$.

(iv) All weak bi-Stirlitz tracks $\gamma$ of $L_{m, n}$ with index $\left(m^{\prime}, n^{\prime}\right)$ such that $m^{\prime}+n^{\prime}=$ $m+n$ have trace either $\left(\tilde{\sigma}_{0}, \tilde{\tau}_{0}\right)$ or $\left(\tilde{\tau}_{0}, \tilde{\sigma}_{0}\right)$ (see Definition 11.2).

Proof. (i)-(iii) are straightforward. The result of (iv) follows easily from Lemma10.4

The proof of the following lemma is straightforward.

Lemma 11.4. Let $K$ and $L$ be lattices, let $f: K \rightarrow L$ be a lower bounded, surjective lattice homomorphism, let $\beta: L \hookrightarrow K$ be the join-homomorphism defined by $\beta(x)=$ $\min f^{-1}\{x\}$, for all $x \in L$. Then the following statements hold:

(i) The image under $\beta$ of $\mathrm{J}(L)$ is contained in $\mathrm{J}(K)$.

(ii) The image under $\beta$ of any minimal nontrivial join-cover of $L$ is a minimal nontrivial join-cover of $K$.

(iii) The image under $\beta$ of any Stirlitz track (resp., bi-Stirlitz track) of $L$ is a Stirlitz track (resp., bi-Stirlitz track) of $K$.

Now we can classify all finite subdirectly irreducible members of $\mathbf{S U B}(\mathcal{L O})$.

\section{Theorem 11.5.}

(i) Let $L$ be a finite subdirectly irreducible lattice in $\mathbf{S U B}(\mathcal{L O})$, let $n$ be a positive integer. Then either $\mathbf{C o}(n)$ embeds into $L$ or $L$ embeds into $\mathbf{C o}(n)$.

(ii) Let $\mathbf{V}$ be a subvariety of $\mathbf{S U B}(\mathcal{L} \mathcal{O})$. Then either $\mathbf{S U B}(n) \subseteq \mathbf{V}$ or $\mathbf{V} \subseteq$ $\operatorname{SUB}(n)$, for every positive integer $n$.

(iii) The only finite subdirectly irreducible members of $\mathbf{S U B}(\mathcal{L O})$ are the $\mathbf{C o}(n)$, for $n>0$, and the $L_{m, n}$, for $m, n>0$. 
Proof. (i) Suppose that $L$ does not embed into $\mathbf{C o}(n)$. Since $L$ is subdirectly irreducible, it does not belong to $\mathbf{S U B}(n)$, thus, by Theorem 9.4 it does not belong to $\mathbf{S U B}_{n-1}$. Hence, there exists $k \in\{1, \ldots, n-1\}$ such that $L$ does not satisfy the identity $\left(\mathrm{H}_{k, n-k}\right)$, see [13. Since $L$ is finite, it follows from [13. Proposition 6.2] that $L$ has a bi-Stirlitz track of index $(k, n-k)$, thus, by Lemma 10.4 $\mathbf{C o}(n)$ embeds into $L$.

(ii) Suppose that $\mathbf{S U B}(n)$ is not contained in $\mathbf{V}$, that is, $\mathbf{C o}(n) \notin \mathbf{V}$. We prove that any lattice $L \in \mathbf{V}$ belongs to $\mathbf{S U B}(n)$. Since $\mathbf{S U B}(\mathcal{L O})$ is locally finite (Theorem [9.5), it suffices to consider the case where $L$ is finite, hence it suffices to consider the case where $L$ is finite and subdirectly irreducible. From $\mathbf{C o}(n) \notin \mathbf{V}$ it follows that $\mathbf{C o}(n)$ does not embed into $L$, thus, by (i), $L$ embeds into $\mathbf{C o}(n)$, thus it belongs to $\mathbf{S U B}(n)$.

(iii) Let $L$ be a finite subdirectly irreducible member of $\mathbf{S U B}(\mathcal{L O})$. Suppose that $L$ is nondistributive. There exists a largest integer $n \geq 2$ such that $\mathbf{C o}(n)$ embeds into $L$. By (i), $L$ embeds into $\mathbf{C o}(n+1)$. Suppose that $L$ is not isomorphic to $\mathbf{C o}(n)$. Since $L$ is subdirectly irreducible, $L \notin \mathbf{S U B}(n)$, thus, as in the proof of (i), there are $k, l>0$ such that $k+l=n$ and $L$ does not satisfy $\left(\mathrm{H}_{k, l}\right)$, hence $L$ has a bi-Stirlitz track $(\sigma, \tau)$ of index $(k, l)$, with, say,

$$
\begin{aligned}
& \sigma=\left(\left\langle X_{i} \mid 0 \leq i \leq k\right\rangle,\left\langle X_{i}^{\prime} \mid 1 \leq i \leq k\right\rangle\right), \\
& \tau=\left(\left\langle Y_{j} \mid 0 \leq j \leq l\right\rangle,\left\langle Y_{j}^{\prime} \mid 1 \leq j \leq l\right\rangle\right) .
\end{aligned}
$$

Put $Z=X_{0}=Y_{0}$. It follows from Lemma 10.4 that, up to possibly reversing the ordering of $n+1$ or exchanging $\sigma$ and $\tau$,

$$
\begin{gathered}
X_{k} \triangleleft \cdots \triangleleft X_{1} \triangleleft Y_{1} \triangleleft \cdots \triangleleft Y_{l} \text { and } X_{1} \unlhd_{\mathrm{w}} Z \unlhd_{\mathrm{w}} Y_{1}, \\
X_{1} \vee Y_{1} \neq X_{1} \cup Y_{1} \text { and } Z \cap Y_{1}=\varnothing .
\end{gathered}
$$

Since $L$ has at most $n+1$ join-irreducible elements, these elements are exactly the $X_{i}$-s, for $1 \leq i \leq k$, the $Y_{j}$-s, for $1 \leq j \leq l$, and $Z$. Furthermore, it follows from (11.1) and (11.2) that $X_{i}=\{k-i\}$ for $1 \leq i \leq k, Y_{j}=\{k+j\}$ for $1 \leq j \leq l$, and $Z$ is either equal to $\{k\}$ or to $\{k-1, k\}$. In the first case, $L \cong \mathbf{C o}(n+1)$, a contradiction, thus the second case applies. But then, $L \cong L_{k, l}$.

Remark 11.6. There exists a proper class of infinite subdirectly irreducible lattices in $\operatorname{SUB}(\mathcal{L O})$, for example, all lattices of the form $\mathbf{C o}(T)$ where $T$ is an infinite chain. However, each of those lattices generates the variety $\mathbf{S U B}(\mathcal{L O})$.

As the union of the $\mathbf{S U B}(n)$, for $1 \leq n<\omega$, generates $\mathbf{S U B}(\mathcal{L O})$, we obtain the following corollary.

Corollary 11.7. Every proper subvariety of $\mathbf{S U B}(\mathcal{L O})$ is finitely generated.

For a lattice $L$, let $\mathbf{V}(L)$ denote the lattice variety generated by $L$.

Proposition 11.8. Let $(m, n)$ and $\left(m^{\prime}, n^{\prime}\right)$ be pairs of positive integers such that $m+n=m^{\prime}+n^{\prime}$. If $L_{m, n}$ belongs to $\mathbf{V}\left(L_{m^{\prime}, n^{\prime}}\right)$, then $(m, n)=\left(m^{\prime}, n^{\prime}\right)$.

Proof. By Jónsson's Lemma (see B. Jónsson [9] or P. Jipsen and H. Rose 7]), there are a sublattice $L$ of $L_{m^{\prime}, n^{\prime}}$ and a congruence $\theta$ of $L$ such that $L_{m, n} \cong L / \theta$. The canonical bi-Stirlitz track $\left(\sigma_{0}, \tau_{0}\right)$ of index $(m, n)$ of $L_{m, n} \cong L / \theta$ can be, by 
Lemma 11.4 lifted to a bi-Stirlitz track $(\sigma, \tau)$ of index $(m, n)$ of $L$, say,

$$
\begin{array}{ll}
\sigma=\left(\left\langle x_{i} \mid 0 \leq i \leq m\right\rangle,\left\langle x^{\prime} \mid 1 \leq i \leq m\right\rangle\right), & \text { for some } x^{\prime} \in \mathrm{J}(L), \\
\tau=\left(\left\langle y_{j} \mid 0 \leq j \leq n\right\rangle,\left\langle y^{\prime} \mid 1 \leq j \leq n\right\rangle\right), & \text { for some } y^{\prime} \in \mathrm{J}(L),
\end{array}
$$

with the additional property

$$
x_{1}<x_{0}
$$

(because $\left(\sigma_{0}, \tau_{0}\right)$ has this property and the map $\beta$ of Lemma 11.4 is an orderembedding). By Lemma $10.2(\bar{\sigma}, \bar{\tau})$ is a weak bi-Stirlitz track of $L$, thus of $L_{m^{\prime}, n^{\prime}}$, of index $(m, n)$, thus, by Lemma 11.3(iv), its trace is either $\left(\tilde{\sigma}_{0}, \tilde{\tau}_{0}\right)$ or $\left(\tilde{\tau}_{0}, \tilde{\sigma}_{0}\right)$. But by (11.3), only the first case is possible, whence $(m, n)=\left(m^{\prime}, n^{\prime}\right)$.

Corollary 11.9. For any integer $n \geq 2$, the lattice $\boldsymbol{B}_{n}$ of all lattice varieties $\mathbf{V}$ such that $\mathbf{S U B}(n) \subseteq \mathbf{V} \subset \mathbf{S U B}(n+1)$ is isomorphic to $\mathbf{2}^{n-1}$.

Proof. It follows from Theorem 11.5 that the join-irreducible elements of $\boldsymbol{B}_{n}$ are exactly the varieties $\mathbf{V}\left(L_{k, l}\right)$, where $k, l>0$ and $k+l=n$. Furthermore, by Proposition 11.8 these varieties are mutually incomparable, hence they are atoms of $\boldsymbol{B}_{n}$. Since $\boldsymbol{B}_{n}$ is finite distributive, it is Boolean with $n-1$ atoms.

The results of this section describe completely the lattice of all subvarieties of $\operatorname{SUB}(\mathcal{L O})$. This lattice is countable. Its bottom is diagrammed on the left half of Figure 4 . We use standard notation, for example, $\mathbf{N}_{5}$ denotes the variety generated by the pentagon, $\mathbf{L}_{1,2}$ denotes the variety generated by $L_{1,2}$, and so on. The right half of Figure 4 represents small subdirectly irreducible members of $\mathbf{S U B}(\mathcal{L O})$.

\section{Projective members of $\operatorname{SUB}(\mathcal{L} \mathcal{O})$}

Notation 12.1. Let $m, n>0$. We define lattice-theoretical statements $\Lambda_{n}\left(x_{0}, \ldots, x_{n-1}\right)$ and $\Lambda_{m, n}\left(x_{0}, x_{1}, \ldots, x_{m+n}\right)$ as follows:

$$
\begin{aligned}
\Lambda_{n}\left(x_{0}, \ldots, x_{n-1}\right) \rightleftharpoons & x_{k} \leq x_{i} \vee x_{j} \text { if } 0 \leq i<k<j<n \\
& \text { and } x_{i} \wedge x_{j}=x_{0} \wedge x_{1} \text { for } i \neq j ; \\
\Lambda_{m, n}\left(x_{0}, x_{1}, \ldots, x_{m+n}\right) \rightleftharpoons & x_{k} \leq x_{i} \vee x_{j} \text { if } 0 \leq i<k<j \leq m+n, \\
& x_{m-1} \leq x_{m}, \\
& \text { and } x_{i} \wedge x_{j}=x_{0} \wedge x_{2} \text { for } i \neq j \text { and }\{i, j\} \neq\{m-1, m\} .
\end{aligned}
$$

We leave to the reader the easy proof of the following lemma.

Lemma 12.2. Let $(T, \unlhd)$ be a finite chain, let $n>0$, let $A_{0}, \ldots, A_{n-1}$ be pairwise disjoint elements of $\mathbf{C o}(T)$ such that $A_{k} \subseteq A_{i} \vee A_{j}$, for $0 \leq i<k<j<n$. Then either the following statement or its dual holds:

There are elements $x_{i}, y_{i}(i<n)$ of $T$ such that $A_{i}=\left[x_{i}, y_{i}\right)$, for all $i<n$, and

$$
x_{0} \unlhd y_{0} \unlhd x_{1} \unlhd \cdots \unlhd x_{n-1} \unlhd y_{n-1} .
$$

The following lemma is the key to all projectivity results of the present section.

Lemma 12.3. Let $L \in \mathbf{S U B}(\mathcal{L} \mathcal{O})$. The following statements hold:

(i) For all $n>0$ and all $a_{0}, \ldots, a_{n-1} \in L$ such that $\Lambda_{n}\left(a_{0}, \ldots, a_{n-1}\right)$ holds, there exists a unique $\varphi: \mathbf{C o}(n) \rightarrow L$ such that $\varphi(\{i\})=a_{i}$, for all $i<n$. 


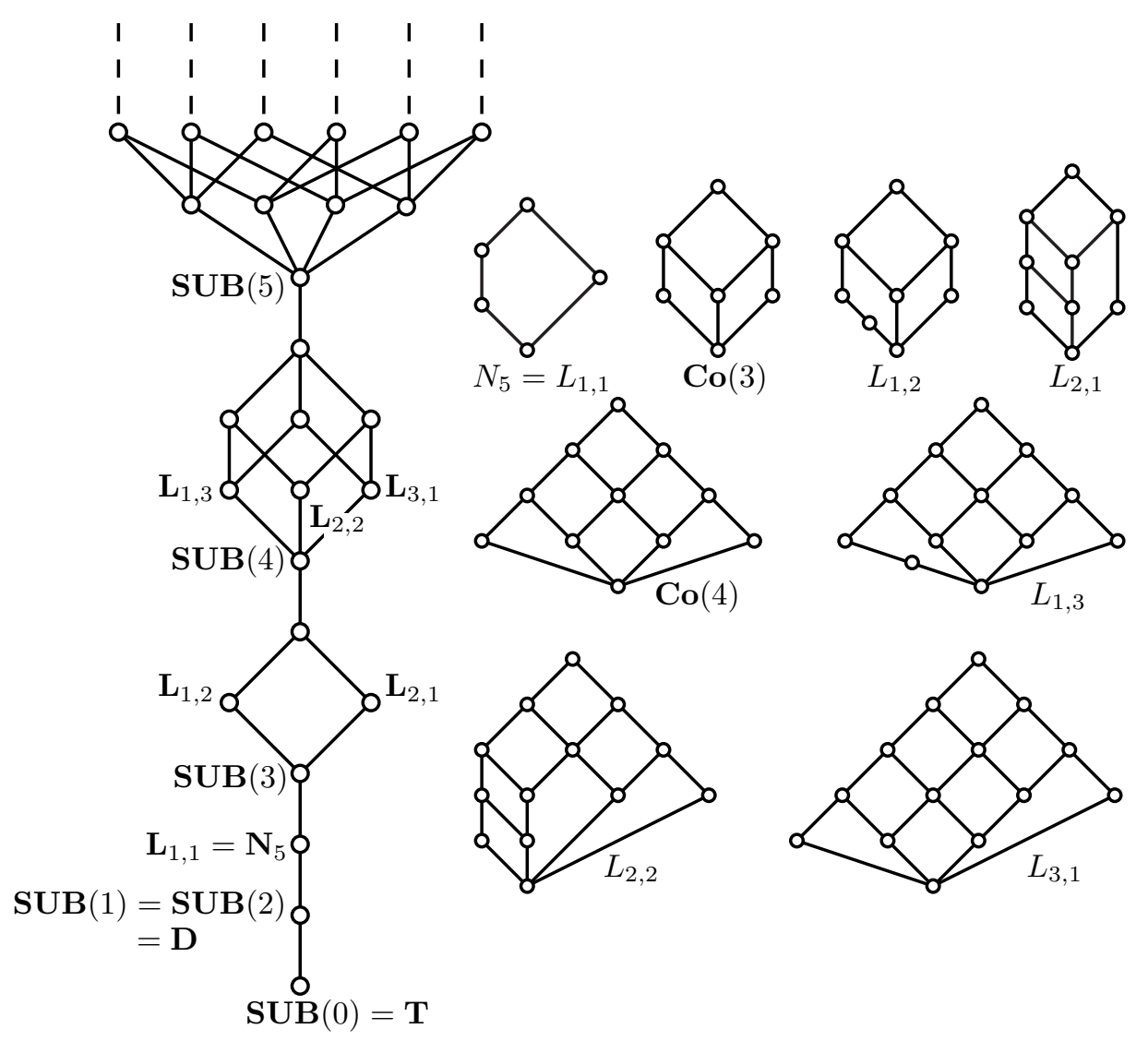

Figure 4. Small subvarieties of $\mathbf{S U B}(\mathcal{L O})$

(ii) For all $m, n>0$ and all $a_{0}, a_{1}, \ldots, a_{m+n} \in L$ such that $\Lambda_{m, n}\left(a_{0}, a_{1}, \ldots, a_{m+n}\right)$ holds, there exists a unique $\varphi: L_{m, n} \rightarrow L$ such that $\varphi(\{i\})=a_{i}$, for all $i \neq m$, while $\varphi(\{m-1, m\})=a_{m}$.

Proof. Without loss of generality, $L$ is generated by $\left\{a_{i} \mid 0 \leq i<n\right\}$ in (i), by $\left\{a_{i} \mid 0 \leq i \leq m+n\right\}$ in (ii). In particular, by Theorem 9.5 $L$ is finite. Since $L$ is a finite member of $\mathbf{S U B}(\mathcal{L} \mathcal{O})$, we may assume, by Theorem 8.2 that $L=\mathbf{C o}(T)$, for a finite chain $(T, \unlhd)$. Let $u$ be the common value for all $a_{i} \wedge a_{j}$ for $i \neq j$ in (i), for $i \neq j$ and $\{i, j\} \neq\{m-1, m\}$ in (ii). The uniqueness statement about $\varphi$ is, in both cases, obvious, and if there is a map $\varphi$ as desired, then it is given by the rule $\varphi(X)=\bigvee_{i \in X} a_{i}$, for all $X \in \mathbf{C o}(n)$ in (i), for all $X \in L_{m, n}$ in (ii), with the convention that the empty join equals $u$. From the assumption that the $a_{i}$-s satisfy (the statement involving joins in) $\Lambda_{n}$ in (i) and $\Lambda_{m, n}$ in (ii) it follows easily that $\varphi$ is a join-homomorphism.

Now we prove that $\varphi$ is a meet-homomorphism. Suppose first that $u$ is nonempty. The join of any two members of $L$ is their union, whence $L$ is distributive. The statement that $\varphi$ is a meet-homomorphism follows immediately in (i). In (ii), for 
all $X, Y \in L_{m, n}$, we compute:

$$
\begin{aligned}
\varphi(X) \wedge \varphi(Y) & =\bigvee\left\langle a_{i} \wedge a_{j} \mid(i, j) \in X \times Y\right\rangle \\
& = \begin{cases}\varphi(X \cap Y) \vee a_{m-1}, & \text { if }(m-1, m) \in(X \times Y) \cup(Y \times X), \\
\varphi(X \cap Y), & \text { otherwise. }\end{cases}
\end{aligned}
$$

But in the first case, $m-1$ belongs to $X \cap Y$, so we obtain again that $\varphi(X) \wedge \varphi(Y)=$ $\varphi(X \cap Y)$.

Suppose now that $u=\varnothing$. By Lemma 12.2 we may assume without loss of generality that $a_{i}=\left[x_{i}, y_{i}\right)$, for elements $x_{i} \unlhd y_{i}$ of $T$, for $i<n$ in (i) and $i \leq m+n$ in (ii), such that

in (i), while

$$
x_{0} \unlhd y_{0} \unlhd \cdots \unlhd x_{n-1} \unlhd y_{n-1}
$$

$$
x_{0} \unlhd y_{0} \unlhd \cdots \unlhd x_{m-2} \unlhd y_{m-2} \unlhd x_{m} \unlhd y_{m} \unlhd \cdots \unlhd x_{m+n} \unlhd y_{m+n}
$$

in (ii). Furthermore, from the assumption on the $a_{i}$-s it follows that $x_{m-1}=x_{m}$ and $y_{m-1} \leq y_{m}$ in (ii), in particular,

$$
x_{0} \unlhd x_{1} \unlhd \cdots \unlhd x_{m+n} \text { and } y_{0} \unlhd y_{1} \unlhd \cdots \unlhd y_{m+n} .
$$

Hence, in both cases (i) and (ii), the value of $\varphi(X)$ for $X$ in the domain of $\varphi$ can be computed by the rule $\varphi(X)=\left[x_{i}, y_{j}\right)$ whenever $X=[i, j]$, for $i \leq j$. It follows easily that $\varphi$ is a meet-homomorphism.

Now we can prove the main result of the present section.

Theorem 12.4. Every finite subdirectly irreducible member of $\mathbf{S U B}(\mathcal{L O})$ is projective in $\mathbf{S U B}(\mathcal{L O})$.

Proof. We first prove that $\mathbf{C o}(n)$ is projective in $\mathbf{S U B}(\mathcal{L O})$, for all $n>0$. Let $L \in \mathbf{S U B}(\mathcal{L}(\mathcal{O})$, let $\pi: L \rightarrow \mathbf{C o}(n)$ be a surjective lattice homomorphism, we prove that there exists a lattice homomorphism $\varphi: \mathbf{C o}(n) \rightarrow L$ such that $\pi \circ \varphi=\operatorname{id}_{\mathbf{C o}(n)}$. Since $\mathbf{C o}(n)$ is finite, we may replace $L$ by a finitely generated sublattice, which, by Theorem 9.5 is finite. Since $L$ is finite, the sublattice $\pi^{-1}\{X\}$ has a least element, that we denote by $\beta(X)$, for any $X \in \mathbf{C o}(n)$. Put $a_{i}=\beta(\{i\})$, for all $i<n$. Since $\beta$ is a join-homomorphism, the following statement holds:

$$
a_{k} \leq a_{i} \vee a_{j} \text {, for } 0 \leq i<k<j<n .
$$

Now we define inductively elements $b^{l}$ and $a_{i}^{l}$ of $L$, for $i<n$ and $l<\omega$, as follows:

$$
\begin{aligned}
a_{i}^{0} & =a_{i} ; \\
b^{l} & =\bigvee\left\langle a_{i}^{l} \wedge a_{j}^{l} \mid i \neq j\right\rangle ; \\
a_{i}^{l+1} & =a_{i}^{l} \vee b^{l} .
\end{aligned}
$$

Since $L$ is finite, there exists $l<\omega$ such that $a_{i}^{l+1}=a_{i}^{l}$, for all $i<n$. From (12.1), (12.2), and (12.4), it is easy to prove, by induction on $l$, the inequalities

$$
a_{k}^{l} \leq a_{i}^{l} \vee a_{j}^{l}, \text { for } 0 \leq i<k<j<n .
$$

Furthermore, for $i \neq j$ and $k$ in $\{0, \ldots, n-1\}, a_{i}^{l} \wedge a_{j}^{l} \leq b^{l} \leq a_{k}^{l+1}=a_{k}^{l}$. Hence, the statement $\Lambda_{n}\left(a_{0}^{l}, \ldots, a_{n-1}^{l}\right)$ holds, thus, by Lemma 12.3(i), there exists a lattice homomorphism $\varphi: \mathbf{C o}(n) \rightarrow L$ such that $\varphi(\{i\})=a_{i}^{l}$, for all $i<n$. From (12.2)(12.4) it follows that $\pi\left(a_{i}^{l}\right)=\{i\}$, for all $i<n$, whence $\pi \circ \varphi=\operatorname{id}_{\mathbf{C o}(n)}$. 
The proof that $L_{m, n}$ is projective, for $m, n>0$, is similar, by using Lemma12.3(ii). The definitions of the $a_{i}$-s and the $a_{i}^{l}$-s are exactly the same as for the $\mathbf{C o}(n)$ case, while the join in the definition of $b^{l}$ in (12.3) has to be taken over the pairs $(i, j)$ such that $i \neq j$ and $\{i, j\} \neq\{m-1, m\}$.

By Theorem 11.5 there are no other finite subdirectly irreducible members of $\operatorname{SUB}(\mathcal{L O})$, thus the proof is complete.

As a consequence of this, we obtain the following result, which shows that $\operatorname{SUB}(\mathcal{L O})$ is a quite peculiar variety, see the contrast with Example 14.1

Theorem 12.5. Every subquasivariety of $\mathbf{S U B}(\mathcal{L O})$ is a variety.

Proof. Let $\mathbf{Q}$ be a subquasivariety of $\mathbf{S U B}(\mathcal{L} \mathcal{O})$, we prove that $\mathbf{Q}$ is a variety. It suffices to prove that every homomorphic image $L$ of a lattice $L^{\prime}$ in $\mathbf{Q}$ belongs to $\mathbf{Q}$. Since $L$ belongs to the locally finite variety $\operatorname{SUB}(\mathcal{L O})$, it suffices to consider the case where $L$ is finite. By considering the subdirect decomposition of $L$, it suffices then to consider the case where $L$ is subdirectly irreducible. By Theorem 12.4 $L$ is projective within $\mathbf{S U B}(\mathcal{L O})$, thus it embeds into $L^{\prime}$; whence $L$ belongs to $\mathbf{Q}$.

\section{An EXAmple}

For a chain $Q$ and a subset $P$ of $Q$, endowed with the induced ordering, the lattice $\mathbf{C o}(P)$ embeds into $\mathbf{C o}(Q)$, thus it belongs to the variety generated by $\mathbf{C o}(Q)$. We shall now show, through an example, that this simple observation cannot be extended to arbitrary posets.

Let $P$ and $Q$ be the posets diagrammed on Figure 5. Obviously, $P$ is a subset of $Q$, endowed with the induced ordering.

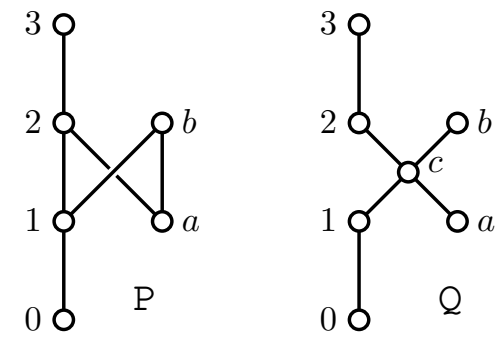

Figure 5. The posets $P$ and $Q$

By induction on the natural number $n$, we define lattice terms $x_{1}^{(n)}, x_{2}^{(n)}, s$, and $t$, in the variables $x_{0}, x_{1}, x_{2}, x_{3}, x_{a}, x_{b}$, putting $x_{1}^{(0)}=x_{1}, x_{2}^{(0)}=x_{2}$, and

$$
\begin{aligned}
& x_{1}^{(n+1)}=x_{1}^{(n)} \wedge\left(x_{0} \vee x_{2}^{(n)}\right) \wedge\left(x_{0} \vee x_{b}\right) ; \\
& x_{2}^{(n+1)}=x_{2}^{(n)} \wedge\left(x_{3} \vee x_{1}^{(n)}\right) \wedge\left(x_{3} \vee x_{a}\right),
\end{aligned}
$$

for all $n<\omega$, then $s=x_{1} \wedge\left(x_{0} \vee\left(\left(x_{1} \vee x_{b}\right) \wedge\left(x_{2} \vee x_{a}\right)\right)\right)$, and then

$$
\begin{aligned}
t=\left(x_{1} \wedge x_{b}\right) & \vee\left(x_{1} \wedge\left(x_{0} \vee x_{a}\right)\right) \vee\left(x_{1} \wedge\left(x_{2} \vee x_{a}\right)\right) \vee\left(x_{1} \wedge\left(x_{0} \vee\left(x_{2} \wedge\left(x_{1} \vee x_{a}\right)\right)\right)\right) \\
& \vee\left(x_{1} \wedge\left(x_{0} \vee\left(x_{2} \wedge\left(x_{1} \vee x_{b}\right)\right)\right)\right) \vee\left(x_{1} \wedge\left(x_{0} \vee\left(x_{2} \wedge\left(x_{3} \vee x_{b}\right)\right)\right)\right) .
\end{aligned}
$$


Finally, let $(*)$ be the following identity:

$$
x_{1}^{(2)} \leq s \vee t .
$$

Lemma 13.1. The lattice $\mathbf{C o}(Q)$ satisfies (*).

Proof. Let $X_{0}, X_{1}, X_{2}, X_{3}, X_{a}, X_{b}$ be elements of $\mathbf{C o}(Q)$, let $S$ and $T$ be obtained by evaluating $s$ and $t$ at those parameters. We prove that $X_{1}^{(2)}$ is a subset of $S \cup T$. So, let $x_{1} \in X_{1}^{(2)}$. If $x_{1} \in X_{0} \cup X_{2}$, then $x_{1} \in\left(X_{0} \cap X_{1}\right) \cup\left(X_{2} \cap X_{1}\right) \subseteq T$; suppose now that $x_{1} \notin X_{0} \cup X_{2}$. Since $x_{1} \in X_{0} \vee X_{2}^{(1)}$, there are $x_{0} \in X_{0}$ and $x_{2} \in X_{2}^{(1)}$ such that either $x_{0} \triangleleft x_{1} \triangleleft x_{2}$ or $x_{2} \triangleleft x_{1} \triangleleft x_{0}$.

Suppose that the first case occurs. If $x_{2} \in X_{1} \cup X_{3}$, then

$$
x_{1} \in\left(X_{1} \cap\left(X_{0} \vee\left(X_{1} \cap X_{2}\right)\right)\right) \cup\left(X_{1} \cap\left(X_{0} \vee\left(X_{2} \cap X_{3}\right)\right)\right) \subseteq T .
$$

Suppose now that $x_{2} \notin X_{1} \cup X_{3}$. Since $x_{2} \in X_{1} \vee X_{3}$, there are $x \in X_{1}$ and $x_{3} \in X_{3}$ such that either $x \triangleleft x_{2} \triangleleft x_{3}$ or $x_{3} \triangleleft x_{2} \triangleleft x$. In the second case, from $x_{1} \triangleleft x_{2} \triangleleft x$ it follows that $x_{2} \in X_{1}$, a contradiction. Thus $x_{2} \triangleleft x_{3}$.

From $x_{1} \in X_{1}^{(2)}$ it follows that $x_{1} \in X_{0} \vee X_{b}$. If $x_{1}$ belongs to $\downarrow X_{0}$ (the lower subset of $Q$ generated by $X_{0}$ ), then $x_{1} \in X_{0} \cap X_{1} \subseteq T$. If $x_{1} \in X_{b}$, then $x_{1} \in$ $X_{1} \cap X_{b} \subseteq T$. Suppose that $x_{1} \notin \downarrow X_{0} \cup X_{b}$. Since $x_{1} \in X_{0} \vee X_{b}$, there exists $x_{b} \in X_{b}$ such that $x_{1} \triangleleft x_{b}$. Furthermore, from $x_{2} \in X_{2}^{(1)}$ it follows that $x_{2} \in X_{3} \vee X_{a}$. If $x_{2} \in \downarrow X_{a}$, then $x_{2} \in X_{2} \cap\left(X_{1} \vee X_{a}\right)$, thus $x_{1} \in X_{1} \cap\left(X_{0} \vee\left(X_{2} \cap\left(X_{1} \vee X_{a}\right)\right)\right) \subseteq T$. Suppose now that $x_{2} \notin \downarrow X_{a}$. Since $x_{2} \notin X_{3}$ and $x_{2} \in X_{3} \vee X_{a}$, there exists $x_{a} \in X_{a}$ such that $x_{a} \triangleleft x_{2}$.

If $x_{a} \unlhd x_{1}$, then $x_{1} \in X_{1} \cap\left(X_{2} \vee X_{a}\right) \subseteq T$. If $x_{1} \unlhd x_{a}$, then $x_{1} \in X_{1} \cap\left(X_{0} \vee X_{a}\right) \subseteq$ $T$. Suppose now that $x_{a} \| x_{1}$ (where $\|$ denotes incomparability). If $x_{2} \unlhd x_{b}$, then $x_{2} \in X_{2} \cap\left(X_{1} \vee X_{b}\right)$, thus $x_{1} \in X_{1} \cap\left(X_{0} \vee\left(X_{2} \cap\left(X_{1} \vee X_{b}\right)\right)\right) \subseteq T$. If $x_{b} \unlhd x_{2}$, then $x_{2} \in X_{2} \cap\left(X_{3} \vee X_{b}\right)$, thus $x_{1} \in X_{1} \cap\left(X_{0} \vee\left(X_{2} \cap\left(X_{3} \vee X_{b}\right)\right)\right) \subseteq T$. Suppose now that $x_{2} \| x_{b}$. Since $x_{1} \| x_{a}$, we have obtained the inequalities

$$
x_{0} \triangleleft x_{1} \triangleleft x_{2} \triangleleft x_{3}, \quad x_{1} \triangleleft x_{b}, \quad x_{a} \triangleleft x_{2}, \quad x_{1} \| x_{a}, \quad \text { and } \quad x_{2} \| x_{b} .
$$

This leaves the only possibility $x_{p}=p$, for all $p \in P$. In particular,

$$
x_{1}=1 \in\{0\} \vee\{c\} \subseteq X_{0} \vee\left(\left(X_{1} \vee X_{b}\right) \cap\left(X_{2} \vee X_{a}\right)\right),
$$

from which it follows that $x_{1} \in S$.

The other case to consider is $x_{2} \triangleleft x_{1} \triangleleft x_{0}$. Then, applying the argument above to the dual of $\triangleleft$, we obtain the dual of (13.1), whence $x_{k}=3-k$, for all $k \in\{0,1,2,3\}, x_{a}=b$, and $x_{b}=a$. In particular,

$$
x_{1}=2 \in\{3\} \vee\{c\} \subseteq X_{0} \vee\left(\left(X_{1} \vee X_{b}\right) \cap\left(X_{2} \vee X_{a}\right)\right),
$$

from which it follows again that $x_{1} \in S$. In any case, $x_{1} \in S \cup T$.

Lemma 13.2. The lattice $\mathbf{C o}(P)$ does not satisfy (*).

Proof. Put $x_{p}=\{p\}$, an element of $\mathbf{C o}(P)$, for any $p \in P$. Then the left hand side of $(*)$, evaluated with those parameters, is $x_{1}=x_{1}^{(2)}=\{1\}$, while the right hand side is empty. Therefore, $\mathbf{C o}(P)$ does not satisfy $(*)$.

Hence we have reached the desired conclusion. 
Proposition 13.3. The poset $P$ embeds into the finite poset $Q$, but the lattice $\mathbf{C o}(P)$ does not belong to the variety generated by $\mathbf{C o}(Q)$.

\section{Open PROBLEMS}

As in [13, we denote, for a class $\mathcal{K}$ of posets, by $\mathbf{S U B}(\mathcal{K})$ the lattice variety generated by $\{\mathbf{C o}(P) \mid P \in \mathcal{K}\}$. Say that a lattice variety $\mathbf{V}$ is a Stirlitz variety, if it is of the form $\mathbf{S U B}(\mathcal{K})$ for some class $\mathcal{K}$ of posets.

It is clear that any join of Stirlitz varieties is a Stirlitz variety, thus the set of all Stirlitz varieties, partially ordered by inclusion, is a complete join-semilattice. In particular, it is a lattice, however, we do not know whether the meet in this lattice is the same as the meet for varieties.

Problem 1. Is the intersection of two Stirlitz varieties a Stirlitz variety?

Problem 2. Let $L$ be a lattice in SUB. Does there exist a smallest Stirlitz variety $\mathbf{V}$ such that $L \in \mathbf{V}$ ?

A related problem is the following.

Problem 3. For a finite lattice $L$ in SUB, are there only finitely many Stirlitz varieties $\mathbf{V}$ which are minimal with the property that $L \in \mathbf{V}$ ?

Analogies between our results with classical results of the spatial theory of modular lattices may fail. For example, the main result of C. Herrmann, D. Pickering, and M. Roddy [6] states that every modular lattice embeds, within its variety, into an algebraic and spatial modular lattice. On the other hand, every lattice $L$ in SUB embeds into an algebraic and spatial lattice in SUB - namely, some $\mathbf{C o}(P)$, however, $\mathbf{C o}(P)$ may not belong to $\mathbf{V}(L)$, for example for $L=N_{5}$. This leads to the following problem.

Problem 4. Does every lattice in SUB embed, within its variety, into some algebraic and spatial lattice?

Of course, by Whitman's Theorem, every lattice $L$ embeds into a partition lattice, which is both algebraic and spatial, but which does not necessarily lie in the same variety as $L$. We do not even know whether every lattice embeds, within its variety, into an algebraic and spatial lattice! While working on the present paper, the authors met the following intriguing problem.

Problem 5. Can every lattice be embedded into some lattice that is both algebraic and dually algebraic?

Note added. The second author recently solved Problem [5

Problem 6. For a finite poset $P$, is the class of all sublattices of powers of $\mathbf{C o}(P)$ a variety?

The answer to Problem 6 in the particular case where $P$ is a chain is, by the results of the present paper, positive, see also Theorem 12.5. The results of Section 13 also suggest a positive answer to Problem [ 6 in general.

Example 14.1. There are many finite lattices $L$ for which the quasivariety $\mathbf{Q}(L)$ generated by $L$ is not a variety, for example, the modular lattice $M_{3-3}$ of Figure 6 , see V. A. Gorbunov [3 p. 257]. It is also possible to find $L$ a bounded homomorphic image of a free lattice. For example, the lattice $L_{9}^{1}$, see P. Jipsen and H. Rose 

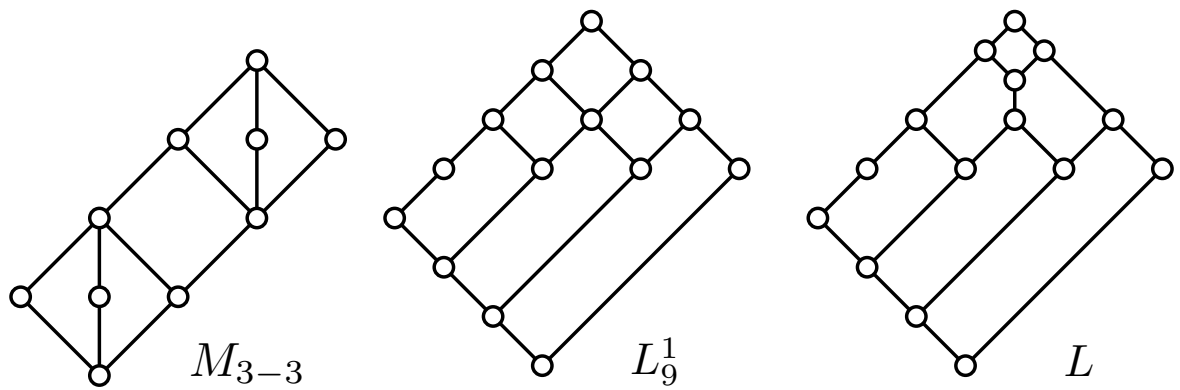

Figure 6 . The lattices $M_{3-3}, L_{9}^{1}$, and $L$

[7. 8, is bounded and subdirectly irreducible. It also has a unique doubly reducible element; doubling this element gives a finite, bounded lattice $L$. Furthermore, $L$ satisfies the Whitman condition, thus it is projective, see [5]. The lattices $L_{9}^{1}$ and $L$ are diagrammed on Figure 6. Since $L_{9}^{1}$ is a quotient of $L$, it belongs to $\mathbf{V}(L)$. If $L_{9}^{1}$ belonged to $\mathbf{Q}(L)$, then, since it is subdirectly irreducible, it would embed into $L$, which is easily seen not to be the case. Therefore, $\mathbf{Q}(L) \neq \mathbf{V}(L)$. Compare this with Theorem 12.5

Problem 7. What are the congruence lattices of lattices in $\operatorname{SUB}(\mathcal{L O})$ ?

Our next problems are related to the variety $\mathbf{C s u b}(\mathbf{D})$ studied by V. Slavík in 14. This variety contains the variety $\mathbf{S U B}(\mathcal{L O})$ studied in the present paper, see the proof of Theorem 9.5 In [14, some properties of the finite subdirectly irreducible members of $\mathbf{C s u b}(\mathbf{D})$ are given, for example, every proper dual ideal is a distributive lattice.

Problem 8. Describe the lattice of subvarieties and classify the finite subdirectly irreducible members of $\mathbf{C s u b}(\mathbf{D})$.

In V. Slavík [15], it is proved that $\mathbf{C s u b}(\mathbf{D})$ has uncountably many subvarieties, but this does not seem to rule out a reasonable classification of finitely generated subvarieties.

Furthermore, it is proved in 14] that $\mathbf{C s u b}(\mathbf{D}) \cap \mathbf{M}=\mathbf{M}_{\boldsymbol{\omega}}$, where $\mathbf{M}$ (resp., $\mathbf{M}_{\boldsymbol{\omega}}$ ) denotes the variety of all modular lattices (resp., the variety generated by the infinite countable lattice $M_{\omega}$ of length two). It is well-known that $\mathbf{M}_{\boldsymbol{\omega}}$ is finitely based, see B. Jónsson [10 or [7 Theorem 3.32]. This suggests the following problems.

Problem 9. Is the variety $\operatorname{Csub}(\mathbf{D})$ finitely based? More generally, if $\mathbf{V}$ is a self-dual, finitely based variety of lattices, is $\mathbf{C s u b}(\mathbf{V})$ finitely based?

Problem 10. Describe $\mathbf{C s u b}(\mathbf{D}) \cap \mathbf{S D}_{\vee}$, where $\mathbf{S D}_{\vee}$ denotes the quasivariety of all join-semidistributive lattices. In particular, is $\mathbf{C s u b}(\mathbf{D}) \cap \mathbf{S D}_{\vee}$ a finitely based quasivariety?

\section{ACKNOWLEDGMENT}

This work was completed during the first author's visit at the University of Caen in March and April 2002, supported by a Young Scientist INTAS fellowship program. The hospitality of the SDAD team can never be forgotten. 
This work was started during the two authors' visit at the Charles University, from October to December 2001. Special thanks are due to Jiř́ Tůma and Václav Slavík.

\section{REFERENCES}

[1] K. V. Adaricheva, V.A. Gorbunov, and V.I. Tumanov, Join-semidistributive lattices and convex geometries, Adv. Math. 173 (2003), 1-49.

[2] G. Birkhoff and M. K. Bennett, The convexity lattice of a poset, Order 2 (1985), 223-242.

[3] V.A. Gorbunov, "Algebraic theory of quasivarieties", (Algebraicheskaya teoriya kvazimnogoobrazij) (Russian) Sibirskaya Shkola Algebry i Logiki. 5. Novosibirsk: Nauchnaya Kniga, 1999. xii +368 p. English translation by Plenum, New York, 1998. xii+298 p.

[4] G. Grätzer, "General Lattice Theory. Second edition", new appendices by the author with B. A. Davey, R. Freese, B. Ganter, M. Greferath, P. Jipsen, H. A. Priestley, H. Rose, E. T. Schmidt, S. E. Schmidt, F. Wehrung, and R. Wille. Birkhäuser Verlag, Basel, 1998. xx+663 p.

[5] R. Freese, J. Ježek, and J. B. Nation, "Free Lattices", Mathematical Surveys and Monographs, 42, Amer. Math. Soc., Providence, 1995. viii+293 p.

[6] C. Herrmann, D. Pickering, and M. Roddy, A geometric description of modular lattices, Algebra Universalis 31, no. 3 (1994), 365-396.

[7] P. Jipsen and H. Rose, "Varieties of Lattices", Lecture Notes in Mathematics 1533, SpringerVerlag, Berlin Heidelberg, 1992. x+162 p.

[8] _ "Varieties of Lattices", Appendix F in 4, 555-574.

[9] B. Jónsson, Algebras whose congruence lattices are distributive, Math. Scand. 21 (1967), 110-121.

[10] _ Equational classes of lattices, Math. Scand. 22 (1968), 187-196.

[11] J. von Neumann, "Continuous geometry", Princeton University Press, Princeton, New Jersey, 1960.

[12] M. Semenova and F. Wehrung, Sublattices of lattices of order-convex sets, I. The main representation theorem, preprint 2002.

[13] _ Sublattices of lattices of order-convex sets, II. Posets of finite length, Internat. J. Algebra Comput., to appear.

[14] V. Slavík, On the variety $\operatorname{Csub}(D)$, Comment. Math. Univ. Carolin. 32, no. 3 (1991), 431434.

[15] _ A note on subvarieties of varieties $\operatorname{Csub}(V)$, Riv. Mat. Pura Appl. 16 (1995), 9-11.

(M. Semenova) Institute of Mathematics of the Siberian Branch of RAS, Acad. KopTYUG PROSP. 4, 630090 Novosibirsk, Russia

E-mail address: semenova@math.nsc.ru

(F. Wehrung) CNRS, UMR 6139, Département de Mathématiques, Université de Caen, 14032 Caen Cedex, France

E-mail address: wehrung@math.unicaen.fr

URL: http://www.math.unicaen.fr/ ${ }^{\sim}$ wehrung 